\title{
Mechanisms Underlying Long-Term Synaptic Zinc Plasticity at Mouse Dorsal Cochlear Nucleus Glutamatergic Synapses
}

\author{
${ }^{\circledR}$ Nathan W. Vogler, ${ }^{1} \bigodot^{\circ}$ Vincent M. Betti, ${ }^{2}{ }^{\oplus}$ Jacob M. Goldberg, ${ }^{2}$ and ${ }^{\circledR}$ Thanos Tzounopoulos $^{1}$ \\ ${ }^{1}$ Pittsburgh Hearing Research Center and Department of Otolaryngology, University of Pittsburgh, Pittsburgh, Pennsylvania 15261, and \\ ${ }^{2}$ Department of Chemistry, Colgate University, Hamilton, New York 13346
}

In many brain areas, such as the neocortex, limbic structures, and auditory brainstem, synaptic zinc is released from presynaptic terminals to modulate neurotransmission. As such, synaptic zinc signaling modulates sensory processing and enhances acuity for discrimination of different sensory stimuli. Whereas sensory experience causes long-term changes in synaptic zinc signaling, the mechanisms underlying this long-term synaptic zinc plasticity remain unknown. To study these mechanisms in male and female mice, we used in vitro and in vivo models of zinc plasticity observed at the zinc-rich glutamatergic dorsal cochlear nucleus (DCN) parallel fiber synapses onto cartwheel cells. High-frequency stimulation of DCN parallel fiber synapses induced LTD of synaptic zinc signaling (Z-LTD), evidenced by reduced zinc-mediated inhibition of EPSCs. Low-frequency stimulation induced LTP of synaptic zinc signaling (Z-LTP), evidenced by enhanced zinc-mediated inhibition of EPSCs. Pharmacological manipulations of Group 1 metabotropic glutamate receptors (G1 mGluRs) demonstrated that G1 mGluR activation is necessary and sufficient for inducing Z-LTD and Z-LTP. Pharmacological manipulations of $\mathrm{Ca}^{2+}$ dynamics indicated that rises in postsynaptic $\mathrm{Ca}^{2+}$ are necessary and sufficient for Z-LTD induction. Electrophysiological measurements assessing postsynaptic expression mechanisms, and imaging studies with a ratiometric extracellular zinc sensor probing zinc release, supported that Z-LTD is expressed, at least in part, via reductions in presynaptic zinc release. Finally, exposure of mice to loud sound caused G1 mGluR-dependent Z-LTD at DCN parallel fiber synapses, thus validating our in vitro results. Together, our results reveal a novel mechanism underlying activity- and experience-dependent plasticity of synaptic zinc signaling.

Key words: auditory; synaptic zinc; synaptic mechanisms; synaptic plasticity

Significance Statement

In the neocortex, limbic structures, and auditory brainstem, glutamatergic nerve terminals corelease zinc to modulate excitatory neurotransmission and sensory responses. Moreover, sensory experience causes bidirectional, long-term changes in synaptic zinc signaling. However, the mechanisms of this long-term synaptic zinc plasticity remain unknown. Here, we identified a novel Group $1 \mathrm{mGluR}$-dependent mechanism that causes bidirectional, long-term changes in synaptic zinc signaling. Our results highlight new mechanisms of brain adaptation during sensory processing, and potentially point to mechanisms of disorders associated with pathologic adaptation, such as tinnitus.

\section{Introduction}

In many brain areas, including the neocortex, limbic structures, and auditory brainstem, synaptic vesicles are loaded with zinc (Danscher and Stoltenberg, 2005; Frederickson et al., 2005). Synaptically released zinc inhibits synaptic and extrasynaptic NMDAR EPSCs, enhances GABAergic IPSCs mediated by

\footnotetext{
Received Jan. 22, 2020; revised May 13, 2020; accepted May 15, 2020.

Author contributions: N.W.V. and T.T. designed research; N.W.V., V.M.B., and J.M.G. performed research; N.W.V. analyzed data; N.W.V. wrote the first draft of the paper; N.W.V., J.M.G., and T.T. edited the paper; N.W.V. and T.T. wrote the paper.

This work was supported by National Institutes of Health Grants F31-DC015924 to N.W.V. and R01-DC007905 to T.T. We thank Dr. Elias Aizenman, Dr. Charles Anderson, and Patrick Cody for helpful discussions.

The authors declare no competing financial interests.

Correspondence should be addressed to Thanos Tzounopoulos at thanos@pitt.edu.

https://doi.org/10.1523/JNEUROSCI.0175-20.2020

Copyright $\odot 2020$ the authors
}

somatostatin-expressing interneurons in mouse auditory cortex, and modulates AMPAR EPSCs in a context-dependent manner (Vogt et al., 2000; Vergnano et al., 2014; Anderson et al., 2015; Kalappa et al., 2015; Kalappa and Tzounopoulos, 2017; Kouvaros et al., 2020). Namely, synaptic zinc inhibits AMPAR EPSCs during baseline synaptic activity via postsynaptic mechanisms but enhances steady-state AMPAR EPSCs during higher frequencies of synaptic stimulation (Kalappa et al., 2015; Kalappa and Tzounopoulos, 2017). The enhancing effect of synaptic zinc on AMPAR EPSCs is short-lasting and is mediated by short-term, zinc-mediated changes in presynaptic glutamatergic neurotransmission (Perez-Rosello et al., 2013; Kalappa and Tzounopoulos, 2017). Thus, synaptic zinc is a major modulator of baseline neurotransmission and shortterm plasticity of glutamatergic synapses. 
In the auditory cortex of awake mice, synaptic zinc enhances the responsiveness (gain) of principal neurons to sound, reduces the gain of interneurons (Anderson et al., 2017), sharpens the sound frequency tuning of principal neurons (Kumar et al., 2019; Kouvaros et al., 2020), and enhances frequency discrimination acuity (Kumar et al., 2019). In the somatosensory cortex, synaptic zinc signaling contributes to whisker-mediated fine texture discrimination (Patrick Wu and Dyck, 2018). These studies support a general role of synaptic zinc signaling in enhancing acuity for sensory stimulus discrimination in cortical circuits.

Synaptic zinc signaling is plastic. Sensory experience bidirectionally modulates the levels of vesicular zinc and synaptic zinc signaling in several sensory brain areas. In the somatosensory cortex, whisker plucking increases zinc levels, whereas whisker stimulation reduces zinc levels (Brown and Dyck, 2002, 2005). In the primary visual cortex, monocular deprivation increases vesicular zinc levels (Dyck et al., 2003). In the retina, optic nerve damage increases zinc levels, which in turn inhibit optic nerve regeneration and promote cell death (Li et al., 2017). In the dorsal cochlear nucleus (DCN), an auditory brainstem nucleus, exposure to loud sound reduces vesicular zinc levels and synaptic zinc signaling (Kalappa et al., 2015). Yet, the cellular and molecular mechanisms underlying the long-term experience-dependent plasticity of synaptic zinc signaling, termed here synaptic zinc plasticity, remain unknown. Elucidating these mechanisms is crucial for understanding how the brain adapts during normal sensory processing, and why it fails to properly adjust in sensory disorders associated with pathologic central adaptation, such as in tinnitus (Auerbach et al., 2014).

To determine the mechanisms of long-term synaptic zinc plasticity, we used electrophysiological, pharmacological, and imaging approaches in the DCN, which contains granule cell endings (parallel fibers [PFs]) with high levels of synaptic zinc (Frederickson et al., 1988; Rubio and Juiz, 1998; Kalappa et al., 2015). We investigated synaptic zinc plasticity mechanisms in vitro, in response to electrical synaptic activation in brain slices, as well as in vivo, in response to loud sound exposure. Our results demonstrate that bidirectional activity-dependent synaptic zinc plasticity is a previously unknown, Group 1 (G1) mGluR-dependent plasticity mechanism at glutamatergic synapses.

\section{Materials and Methods}

Animals and ethical approval

Male or female ICR mice (Envigo) were used in this study. The average (SD) animal age used was P21.8 $\pm 2.6 \mathrm{~d}$. Mice had access to food and water ad libitum. All steps were taken to minimize animals' pain and suffering. All animal procedures were approved by the Institutional Animal Care and Use Committee of the University of Pittsburgh.

\section{Brain slice preparation}

Mice were deeply anesthetized with isoflurane $\left(3 \%\right.$ in $\left.\mathrm{O}_{2}\right)$, then immediately decapitated, and their brains were removed. Brain slices were prepared in ACSF $\left(34^{\circ} \mathrm{C}\right.$ ) containing the following (in $\mathrm{mM}$ ): $130 \mathrm{NaCl}, 3$ $\mathrm{KCl}, 1.2 \mathrm{CaCl}_{2} \cdot 2 \mathrm{H}_{2} \mathrm{O}, 1.3 \mathrm{MgCl}_{2} \cdot 6 \mathrm{H}_{2} \mathrm{O}, 20 \mathrm{NaHCO}_{3}, 3$ HEPES, and 10 D-glucose, saturated with $95 \% \mathrm{O}_{2} / 5 \% \mathrm{CO}_{2}$ (v/v), $\mathrm{pH} 7.25-7.35, \sim 300$ mOsm. Using a Vibratome (VT1200S; Leica Microsystems), coronal brain slices ( $210 \mu \mathrm{m}$ thickness) containing the left DCN were cut, then placed in a chamber containing warm $\left(34^{\circ} \mathrm{C}\right) \mathrm{ACSF}$, and incubated for $60 \mathrm{~min}$ at $34^{\circ} \mathrm{C}$, then at room temperature (no longer than $3 \mathrm{~h}$ ) before beginning electrophysiology experiments. Incubating ACSF was the same as cutting ACSF, except that it was stirred with Chelex 100 resin (Bio-Rad) for $1 \mathrm{~h}$ to remove contaminating zinc, then filtered using Nalgene rapid flow filters lined with polyethersulfone $(0.2 \mu \mathrm{m}$ pore size). After filtering, high-purity $\mathrm{CaCl}_{2} \cdot 2 \mathrm{H}_{2} \mathrm{O}$ and $\mathrm{MgCl}_{2} \cdot 6 \mathrm{H}_{2} \mathrm{O}$ (99.995\%;
Sigma Millipore) were added. All plastic and glassware used for these experiments were washed with $5 \%$ nitric acid.

\section{Electrophysiology}

Whole-cell recordings. DCN slices were transferred to the recording chamber and perfused with ACSF $(1-2 \mathrm{ml} / \mathrm{min})$, maintained at $\sim 34^{\circ} \mathrm{C}$ using an inline heating system (Warner Instruments). Recording ACSF was the same as incubating ACSF (see above), except that it contained $2.4 \mathrm{mM} \mathrm{CaCl} \cdot 2 \mathrm{H}_{2} \mathrm{O}$. Whole-cell recordings from cartwheel cells (CWCs) were performed using glass micropipettes (3-6 MS; Sutter Instruments). CWCs were identified by the presence of complex spikes in cell-attached configuration before break-in or in response to current injections in current-clamp mode after break-in (Zhang and Oertel, 1993; Manis et al., 1994; Tzounopoulos et al., 2004). Recording pipettes were filled with a potassium-based internal solution (except for Fig. 6, see below) containing the following (in $\mathrm{mM}$ ): $113 \mathrm{~K}$-gluconate, 4.5 $\mathrm{MgCl}_{2} \cdot 6 \mathrm{H}_{2} \mathrm{O}, 14$ Tris-phosphocreatine, 9 HEPES, 0.1 EGTA, $4 \mathrm{Na}_{2} \mathrm{ATP}$, 0.3 Tris-GTP, and 10 sucrose ( $\mathrm{pH} 7.25,295 \mathrm{mOsm}$, junction potential $-12 \mathrm{mV})$. For experiments where intracellular solution contained BAPTA $(10 \mathrm{mM})$, internal solution was the same as the potassium-based internal solution, except that it contained $73 \mathrm{mM} \mathrm{K}$-gluconate and 10 $\mathrm{mM}$ BAPTA- $4 \mathrm{~K}^{+}$. For experiments shown in Figure $6 A-D$ measuring NMDAR EPSCs, recordings were performed using a cesium-based internal solution containing the following (in $\mathrm{mM}$ ): $128 \mathrm{Cs}\left(\mathrm{CH}_{3} \mathrm{O}_{3} \mathrm{~S}\right), 10$ HEPES, $4 \mathrm{MgCl}_{2} \cdot 6 \mathrm{H}_{2} \mathrm{O}, 4 \mathrm{Na}_{2} \mathrm{ATP}, 0.3$ Tris-GTP, 10 Tris-phosphocreatine, 1 EGTA, 1 QX-314, and 3 Na-ascorbate (pH 7.25, 300 mOsm, junction potential $-9 \mathrm{mV}$ ). Voltages were not corrected for junction potentials. Recordings were performed using the acquisition software ephus (Suter et al., 2010) and a MultiClamp 700B amplifier (Molecular Devices). Data were sampled at $10 \mathrm{kHz}$ and low-pass-filtered at $4 \mathrm{kHz}$. Series resistance $\left(\mathrm{R}_{\mathrm{s}},<25 \mathrm{M} \Omega\right)$ was not compensated and was monitored during the recording period by delivering $-5 \mathrm{mV}$ voltage steps for $50 \mathrm{~ms}$. $\mathrm{R}_{\mathrm{s}}$ was calculated by dividing the $-5 \mathrm{mV}$ voltage step by the peak current generated immediately after the voltage step. Input resistance $\left(R_{m}\right)$ was monitored and calculated by dividing the $-5 \mathrm{mV}$ voltage step by the difference between the baseline and steady-state hyperpolarized current, then subtracting $R_{s}$. Recordings were not included if $R_{s}$ or $R_{m}$ changed by $>20 \%$ throughout the experiment. EPSCs were evoked using an Isoflex stimulator (A.M.P.I., $0.1 \mathrm{~ms}$ pulses) through a glass ACSFcontaining theta electrode to stimulate the zinc-rich PFs. The average AMPAR EPSC amplitude during the initial baseline period was $370.8 \pm 7.6 \mathrm{pA}$, which was $\sim 50 \%$ of maximum EPSC amplitude. Cells were considered stable during the baseline period if the EPSC amplitude for every minute during the baseline period varied by $<10 \%$ from the overall mean EPSC amplitude during the baseline period (Fujino and Oertel, 2003). For experiments where intracellular solution contained BAPTA (see above), baseline EPSCs were not recorded until at least $20 \mathrm{~min}$ after breaking into whole-cell configuration, to allow for BAPTA diffusion. All EPSCs were recorded in the presence of SR95531 $(20 \mu \mathrm{M}$, $\mathrm{GABA}_{\mathrm{A}} \mathrm{R}$ antagonist) and strychnine ( $1 \mu \mathrm{M}$, GlyR antagonist). AMPAR EPSCs were recorded in voltage-clamp mode at $-70 \mathrm{mV}$. For pairedpulse experiments, the interstimulus interval was $50 \mathrm{~ms}$. NMDAR EPSCs were evoked by a 5 pulse stimulus train $(20 \mathrm{~Hz})$ (Anderson et al., 2015), recorded in voltage-clamp mode at $40 \mathrm{mV}$, and in the presence of DNQX (20 $\mu \mathrm{M}, \mathrm{AMPA} /$ kainate receptor antagonist). Zinc signaling was examined by applying ZX1 (100 $\mu \mathrm{M})$, a fast, high-affinity extracellular zinc chelator (Pan et al., 2011; Anderson et al., 2015). All drugs were always bath-applied.

Induction of plasticity. High-frequency stimulation (HFS) consisted of three trains of $100 \mathrm{~Hz}$ pulses for $1 \mathrm{~s}$, with $10 \mathrm{~s}$ between trains. Neurons were voltage-clamped at $-70 \mathrm{mV}$ during HFS. For experiments measuring NMDAR EPSCs after HFS (see Fig. 6), DNQX $(20 \mu \mathrm{M})$ was added after HFS, then cells were voltage-clamped at $40 \mathrm{mV}$ for the remainder of the experiment to record NMDAR EPSCs. For experiments using ifenprodil, a selective allosteric inhibitor of GluN2B-containing NMDARs (Fig. 6C,D), ZX1 was applied before ifenprodil to chelate extracellular zinc, because zinc affects NMDAR ifenprodil sensitivity (Hansen et al., 2014). The time course of these experiments (Fig. $6 C, D$ ) was similar to the experiments shown in Figure 6A, except that ZX1 was applied with DNQX, after 
the HFS. After obtaining a stable baseline of NMDAR EPSCs, ifenprodil (300 nM, $1 \mu \mathrm{M}, 3 \mu \mathrm{M}$, or $10 \mu \mathrm{M}$ ) was applied, and EPSCs (\% baseline) were measured $10 \mathrm{~min}$ after ifenprodil application. Low-frequency stimulation (LFS) consisted of $5 \mathrm{~Hz}$ pulses for $3 \mathrm{~min}$. During LFS, cells were held at $-80 \mathrm{mV}$ in current-clamp mode (Oliet et al., 1997). To promote mGluRmediated plasticity, all LFS experiments were performed in the presence of APV (50 $\mu \mathrm{M}$, NMDAR antagonist), and with external ACSF containing 4 $\mathrm{mM} \mathrm{CaCl} 2 \cdot 2 \mathrm{H}_{2} \mathrm{O}$ and $4 \mathrm{mM} \mathrm{MgCl} 2 \cdot 6 \mathrm{H}_{2} \mathrm{O}$ (Oliet et al., 1997). The interleaved experiments shown in Figure $4 F$, examining the effect of $50 \mu \mathrm{M}(\mathrm{S})$ 3,5-dihydroxyphenylglycine (DHPG) application, were also performed in these conditions. For normalized EPSCs (\% baseline), EPSC amplitudes were normalized to the average EPSC amplitude during the 5 min baseline period before HFS/LFS, DHPG, ifenprodil, cyclopiazonic acid (CPA), or ZX1 application. To quantify ZX1 potentiation after HFS/LFS, CPA, or DHPG application, EPSC amplitudes were renormalized to the average EPSC amplitude of the new baseline period $5 \mathrm{~min}$ before ZX1 application. ZX1 potentiation or CPA potentiation (shown in bar graphs) was quantified as the percent increase in the average EPSC amplitude during the last $5 \mathrm{~min}$ of ZX1 or CPA application compared with the $5 \mathrm{~min}$ baseline period before ZX1 or CPA application. The exact time points used for analyses are stated in Detailed values and statistical tests for figures.

\section{Imaging of synaptic zinc release using LZ9}

The extracellular ratiometric fluorescent zinc sensor LZ9 was synthesized based on previously published methods (Kalappa et al., 2015). Imaging of synaptic zinc release using LZ9 was performed based on previously published methods (Anderson et al., 2015; Kalappa et al., 2015). After preparation and incubation of DCN slices (described above), slices were transferred to the imaging chamber and perfused with recirculating ACSF maintained at $\sim 34^{\circ} \mathrm{C}$. ACSF contained the slow zinc chelator CaEDTA $(200 \mu \mathrm{M})$ to reduce background fluorescence, LZ9 $(2 \mu \mathrm{M})$, SR95531 $(20 \mu \mathrm{M})$, and strychnine $(1 \mu \mathrm{M})$. LZ9 is composed of a zincinsensitive red fluorophore lissamine rhodamine B linked to a zinc-sensitive green fluorophore (ZP1). Blue illumination excites ZP1; therefore, the fluorescence emission in response to blue light is zinc-sensitive. Green illumination excites lissamine rhodamine, resulting in zinc-insensitive fluorescence. The ratiometric zinc signal (R) is the ratio of these two fluorescent signals (Anderson et al., 2015). To image LZ9 fluorescence, we used an interleaved, multiplexed approach using ephus in which blue and green light-emitting diodes (M470L2 and M530L2, Thorlabs) were synchronized with the exposure times of a CCD camera (Retiga 2000R, QImaging) so that every other frame used either blue or green excitation. The two emission and two excitation colors were separated using a Pinkel filter set (LF488/543/625-3X-A-000, Semrock). Two-channel, multiplexed movies were acquired at a frame rate of $20 \mathrm{~Hz}$, each channel at a frame rate of $10 \mathrm{~Hz}$, using an upright microscope (Olympus BX5) with epifluorescence optics through a $40 \times$ water immersion objective (Olympus). Baseline synaptic zinc release from DCN PFs was evoked with a theta simulating electrode using a stimulus of 50 pulses delivered at $100 \mathrm{~Hz}$. The ratiometric fluorescent response was visualized using a fourth-order, low-pass, 2D Butterworth filter (example images shown in Fig. 6E). For each experiment, we analyzed a square ROI 100 pixels $(\sim 75 \mu \mathrm{m})$ wide, centered around the pixel with the maximum fluorescence increase $0.5-2 \mathrm{~s}$ after stimulation. Thus, all ROIs were the same size. The ratio (R) of ZP1-based fluorescence to lissamine rhodamine-based fluorescence was calculated by dividing the frame-averaged ROI during blue excitation by the same ROI in the subsequent frame acquired with green excitation for each pair of frames. ROIs were quantified in the unfiltered images. To quantify stimulusevoked zinc fluorescence, we calculated the maximum $\Delta \mathrm{R} / \mathrm{R}$ 0.5-2 s after stimulation, the time window containing the initial fluorescence increase (example traces in Fig. 6E). We compared evoked zinc release (elicited by a stimulus of 50 pulses, $100 \mathrm{~Hz}$ ) before and after delivering HFS (three trains of $100 \mathrm{~Hz}$ pulses for $1 \mathrm{~s}$, with $10 \mathrm{~s}$ between trains). Zinc fluorescence $(\Delta \mathrm{R} / \mathrm{R})$ after HFS was normalized to zinc fluorescence before HFS (Fig. $6 F$ ).

\section{Noise exposure}

Noise exposure was performed based on previously published methods (Kalappa et al., 2015). Namely, sham-exposed or noise-exposed (NE) mice were anesthetized initially using 3\% isoflurane, which was then maintained at $1 \%-1.5 \%$. NE mice were exposed for $4 \mathrm{~h}$ to narrow bandpass noise at $116 \mathrm{~dB}$ sound pressure level (SPL), centered at $16 \mathrm{kHz}$ with a $1.6 \mathrm{kHz}$ bandwidth. Noise was presented unilaterally (left ear) through a pipette tip inserted into the left ear canal, with the other end attached to a calibrated speaker (CF-1; Tucker Davis Technologies). Insertion of the pipette tip into the ear canal did not produce a seal. Sham-exposed mice underwent an identical procedure except without any noise exposure. For mice given intraperitoneal injections of (RS)-1-aminoindan1,5-dicarboxylic acid (AIDA, selective G1 mGluR antagonist, $2 \mathrm{mg} / \mathrm{kg}$ ), one injection was given $30 \mathrm{~min}$ before exposure, and a second injection was given $2 \mathrm{~h}$ later. After noise or sham exposure, auditory brainstem responses (ABRs) were collected and mice recovered from anesthesia; then DCN slices were prepared (within $30 \mathrm{~min}$ after exposure).

\section{ABRs}

ABRs were measured based on previously published methods (Kalappa et al., 2015). ABRs were recorded immediately after noise or sham exposure. During ABR measurements, mice were anesthetized using 3\% isoflurane during induction and $1 \%-1.5 \%$ during maintenance. Mice were placed in a sound-attenuating chamber, and temperature was maintained at $\sim 37^{\circ} \mathrm{C}$ using a heating pad. A subdermal electrode was placed at the vertex, the ground electrode placed ventral to the right pinna, and the reference electrode placed ventral to the left pinna (sham-exposed or NE ear). In NE mice, because no ABRs were detected when recording from the exposed (ipsilateral) ear, we recorded ABRs from the nonexposed (contralateral) ear (see Fig. $7 D$ ). For ABR measurements from contralateral ears of NE mice, the reference electrode was placed ventral to the right pinna (contralateral ear) and the ground electrode placed ventral to the left pinna. ABRs were detected in response to $1 \mathrm{~ms}$ click sound stimuli, presented through a pipette tip inserted into the ear canal, with the other end attached to the speaker (CF-1; Tucker Davis Technologies). ABRs were recorded in response to clicks presented in $10 \mathrm{~dB}$ steps, ranging from 0 to $80 \mathrm{~dB}$ SPL; 1 ms clicks were presented at a rate of 18.56/s using System 3 software package from Tucker Davis Technologies, and ABRs were averaged 512 times and filtered using a $300-3000 \mathrm{~Hz}$ bandpass filter. ABR threshold was defined as the lowest stimulus intensity which generated a reliable Wave I in the response waveform. Wave I amplitude was measured as the peak-to-trough amplitude of the first wave in the ABR waveform (latency $\sim 2 \mathrm{~ms}$ ), in response to $80 \mathrm{~dB}$ SPL clicks.

\section{Drugs}

Salts used for ACSF and internal solutions were purchased from Sigma Millipore. The following drugs were purchased from HelloBio: SR95531 hydrobromide, DL-AP5, DNQX disodium salt, ifenprodil, MPEP hydrochloride, LY367385, DHPG, and CPA. BAPTA tetrapotassium salt (BAPTA-4K ${ }^{+}$) was purchased from Santa Cruz Biotechnology. Strychnine hydrochloride was purchased from Abcam. AIDA was purchased from Tocris. ZX1 was purchased from STREM Chemicals.

\section{Statistical analysis}

All data analysis was performed using MATLAB (The MathWorks), Excel (Microsoft), or Prism 7 (GraphPad). For statistical tests within groups, to determine whether HFS, LFS, DHPG, CPA, or ZX1 significantly changed EPSC amplitude, we used paired $t$ tests (for normally distributed data) or Wilcoxon matched-pairs signed rank tests (for nonnormally distributed data). Data were considered normally distributed if they passed the Shapiro-Wilk normality test. For paired tests, we compared the average EPSC amplitude (in pA) of the $5 \mathrm{~min}$ baseline period to the average EPSC amplitude (in pA) during a 5 min period after HFS, LFS, DHPG, CPA, or ZX1. The exact time points that were used for each test are stated in the figure legends. For comparisons between groups, we used unpaired $t$ tests (for normally distributed data) or MannWhitney tests (for non-normally distributed data). All statistical tests were two-tailed. For comparisons between three or more groups, we used ordinary one-way ANOVA with Bonferroni's multiple comparisons test (for normally distributed data) or Kruskal-Wallis test with Dunn's multiple comparisons test (for non-normally distributed data). 
$\mathrm{IC}_{50}$ was calculated using the Hill equation by fitting the dose-response curve with a nonlinear least-squares fit. The $\mathrm{IC}_{50}$ of each fit was compared using the extra sum-of-squares $F$ test. Significance levels are defined as $p<0.05$. Group data are presented as mean \pm SEM, unless otherwise noted.

Detailed values and statistical tests for figures. Figure $1 B$ : Baseline (average of minutes 1-5) versus ZX1 (average of minutes 21-25) (pA): $n=10,{ }^{*} p=0.002$, Wilcoxon matched-pairs signed rank test. Figure $1 C$ : HFS: baseline (average of minutes 1-5) versus after HFS (average of minutes 19-23) (pA): $n=7, t=3.074, \mathrm{df}=6,{ }^{*} p=0.0218$, paired $t$ test; Baseline versus ZX1 (average of minutes 21-25) (pA): $n=7, t=0.1837$, $\mathrm{df}=6$, not significant, $p=0.8603$, paired $t$ test. Control: Baseline versus ZX1 (average of minutes 21-25) (pA): $n=6,{ }^{*} p=0.0313$, Wilcoxon matched-pairs signed rank test. Figure $1 D$ : ZX1 potentiation (\%): Control: $36.45 \pm 6.852 \%, n=6$. HFS: $3.777 \pm 8.838 \%, n=7$. Control versus HFS: $t=2.844, \mathrm{df}=11,{ }^{*} p=0.016$, unpaired $t$ test. Figure $1 E: n=5-8$ : three cells did not remain stable for the entire time course and were included up to minute 35 . Baseline versus minutes 30-49 (pA): $n=8$, $t=2.796, \mathrm{df}=7,{ }^{*} p=0.0267$, paired $t$ test. Minutes $19-23$ versus minutes 45-49 (pA): $n=5, t=1.971, \mathrm{df}=4$, not significant, $p=0.12$, paired $t$ test.

Figure 2A: HFS + APV: Baseline versus after HFS + APV (minutes 19-23): $n=9, t=4.949, \mathrm{df}=8,{ }^{\star} p=0.0011$, paired $t$ test. Baseline versus ZX1 (minutes 21-25): $n=9$, not significant, $p=0.8203$, Wilcoxon matched-pairs signed rank test. ZX1 potentiation (\%): HFS + APV versus HFS: $t=0.04826, \mathrm{df}=14$, not significant, $p=0.9622$. Figure $2 B$ : Baseline versus after HFS (minutes 21-25): $n=6, t=0.41, \mathrm{df}=5$, not significant, $p=0.6988$, paired $t$ test. Figure 2C: HFS + APV, LY367385/ MPEP: Baseline versus after HFS + APV, LY367385/MPEP (minutes 19-23): $n=6, t=3.022, \mathrm{df}=5,{ }^{\star} p=0.0293$, paired $t$ test. Baseline versus ZX1 (minutes 21-25): $n=5, t=3.669, \mathrm{df}=4,{ }^{*} p=0.0214$, paired $t$ test. One cell was included for analysis of EPSCs following HFS but did not remain stable throughout subsequent ZX1 application and was excluded from analysis following ZX1 application. APV, LY367385/ MPEP: Baseline versus ZX1 (minutes 21-25): $n=5, t=3.22$, $\mathrm{df}=4$, ${ }^{*} p=0.0323$, paired $t$ test. Figure $2 D:$ ZX1 potentiation (\%): HFS + APV: $4.28 \pm 6.08 \%, n=9$. HFS + APV, LY367385/MPEP: $36.07 \pm$ 9.05\%, $n=5$. APV, LY367385/MPEP: $33.76 \pm 8.96 \%, n=5$. One-way ANOVA: $F=6.048,{ }^{\star} p=0.0111$. HFS + APV versus HFS + APV, LY367385/MPEP: ${ }^{*} p=0.0181$; HFS + APV, LY367385/MPEP versus APV, LY367385/MPEP: not significant, $p>0.9999$; Bonferroni’s multiple comparisons test.

Figure 3A: LFS: Baseline versus after LFS (minutes 19-23): $n=8$, $t=1.344, \mathrm{df}=7$, not significant, $p=0.2209$, paired $t$ test. Baseline versus ZX1 (minutes 21-25): $n=6, t=5.621, \mathrm{df}=5,{ }^{*} p=0.0025$, paired $t$ test. Two cells were included for analysis of EPSCs following LFS but did not remain stable throughout subsequent ZX1 application and were excluded from analysis following ZX1 application. Control: Baseline versus ZX1 (minutes 21-25): $n=5, t=3.785, \mathrm{df}=4,{ }^{*} p=0.0194$, paired $t$ test. Figure 3B: Baseline versus after LFS (minutes 23-27): $n=5, t=3.419$, df $=4,{ }^{\star} p=0.0268$, paired $t$ test. Figure $3 C$ : LFS + LY367385/MPEP: Baseline versus after LFS (minutes 19-23): $n=6$, $t=0.4227, \mathrm{df}=5$, not significant, $p=0.6901$, paired $t$ test. Baseline versus ZX1 (minutes 21-25): $n=6, t=3.29, \mathrm{df}=5$, ${ }^{*} p=0.0217$, paired $t$ test. LY367385/MPEP: versus ZX1 (minutes 21-25): $n=6, t=3.668$, $\mathrm{df}=5,{ }^{*} p=0.0145$, paired $t$ test. Figure $3 D$ : ZX1 potentiation (\%): Control: $19.65 \pm 4.3 \%, n=5$. LFS: $57.86 \pm 12.4 \%, n=6$. LFS + LY367385/MPEP: $20.19 \pm 7.68 \%, n=6$. LY367385/MPEP: $29.54 \pm$ 9.73\%, $n=6$. One-way ANOVA: $F=3.737,{ }^{\star} p=0.0289$. Control versus LFS: ${ }^{\star} p=0.0334$; LFS versus LFS + LY367385/MPEP: ${ }^{*} p=0.0271$; LFS + LY367385/MPEP versus LY367385/MPEP: not significant, $p>$ 0.999; Bonferroni's multiple comparisons test. Figure $3 E$ : LFS: Minutes 19-23 versus minutes 44-48: $n=4, t=1.186, \mathrm{df}=3$, not significant, $p=0.3211$, paired $t$ test. LFS + LY367385/MPEP: Minutes 19-23 versus minutes 44-48: $n=4, p=0.875$, Wilcoxon matched-pairs signed rank test.

Figure 4A: DHPG $(50 \mu \mathrm{M})$ : Baseline versus minutes 16-20: $n=6$, $t=5.438, \mathrm{df}=5,{ }^{*} p=0.0029$, paired $t$ test. Baseline versus ZX1 (minutes 21-25): $n=5, t=3.717, \mathrm{df}=4,{ }^{*} p=0.0205$, paired $t$ test. One cell was included for analysis of EPSCs following $50 \mu \mathrm{M}$ DHPG but did not remain stable throughout subsequent ZX1 application and was excluded from analysis following ZX1 application. DHPG $(5 \mu \mathrm{M})$ : Baseline versus minutes 16-20: $n=6, t=1.736, \mathrm{df}=5$, not significant, $p=0.1431$, paired $t$ test. Baseline versus ZX1 (minutes 21-25): $n=5, t=0.2619$, $\mathrm{df}=4$, not significant, $p=0.8063$, paired $t$ test. One cell was included for analysis of EPSCs following $5 \mu \mathrm{M}$ DHPG but did not remain stable throughout subsequent ZX1 application and was excluded from analysis following ZX1 application. Figure 4B: ZX1 potentiation (\%): DHPG (50 $\mu \mathrm{M})$ : $93.51 \pm 10.92 \%, n=5$. DHPG $(5 \mu \mathrm{M}): 0.44 \pm 7.08 \%, n=5$. One-way ANOVA: $F=23.19,{ }^{\star} p<0.0001$. Control versus DHPG $(50 \mu \mathrm{M}):{ }^{\star} p=$ 0.0006; Control versus DHPG $(5 \mu \mathrm{M}):{ }^{*} p=0.0285$; Bonferroni's multiple comparisons test. Figure $4 C$ : DHPG $(50 \mu \mathrm{M})$ : Minutes $16-20$ versus minutes 40-44: $n=5, t=1.499, \mathrm{df}=4$, not significant, $p=0.2083$, paired $t$ test. DHPG $(5 \mu \mathrm{M})$ : Minutes 16-20 versus minutes 40-44: $n=4$, $t=1.093, \mathrm{df}=3$, not significant, $p=0.3544$, paired $t$ test. Figure $4 D$ : Baseline versus minutes 16-20: $n=5, t=1.228$, df $=4$, not significant, $p=0.2867$, paired $t$ test. Figure 4E: Baseline versus minutes 16-20: $n=5$, $t=3.494, \mathrm{df}=4,{ }^{*} p=0.025$, paired $t$ test. Figure $4 F$ : Baseline versus minutes 16-20: $n=5, t=2.923, \mathrm{df}=4,{ }^{*} p=0.0431$, paired $t$ test. Baseline versus ZX1 (minutes 21-25): $n=5, t=3.462, \mathrm{df}=4,{ }^{*} p=0.0258$, paired $t$ test. Figure 4G: Baseline versus minutes 20-24: $n=5, t=2.086, \mathrm{df}=4$, not significant, $p=0.1053$, paired $t$ test. Baseline versus ZX1 (minutes 21-25): $n=5, t=5.932, \mathrm{df}=4,{ }^{*} p=0.004$, paired $t$ test. Figure $4 H: \mathrm{ZX} 1$ potentiation (\%): DHPG $(50 \mu \mathrm{M}): 55.83 \pm 17.9 \%, n=5$. LFS + DHPG $(50 \mu \mathrm{M}): 74.65 \pm 17.6 \%, n=5$. One-way ANOVA: $F=0.4126$, not significant, $p=0.6703$. LFS + DHPG $(50 \mu \mathrm{M})$ versus LFS: not significant, $p=0.9181 ; \mathrm{LFS}+$ DHPG $(50 \mu \mathrm{M})$ versus DHPG $(50 \mu \mathrm{M})$ : not significant, $p=0.8553$; Bonferroni's multiple comparisons test.

Figure 5A: Baseline versus HFS (minutes 19-23) (pA): $n=7$, $t=1.1214, \mathrm{df}=6$, not significant, $p=0.2705$, paired $t$ test. Baseline versus ZX1 (minutes 21-25): $n=7, t=5.286, \mathrm{df}=6,{ }^{*} p=0.0019$, paired $t$ test. Figure 5B: Baseline versus ZX1 (minutes 21-25): $n=7, t=4.611, \mathrm{df}=6$, ${ }^{*} p=0.0036$, paired $t$ test. Figure $5 C$ : ZX1 potentiation (\%): HFS (BAPTA): $26.88 \pm 3.325, n=7$. BAPTA: $36.05 \pm 7.998, n=7$. HFS (BAPTA) versus BAPTA: $t=1.059, \mathrm{df}=12$; not significant, $p=0.3106$, unpaired $t$ test. Figure 5D: Control: Baseline versus CPA (minutes 16-20): $n=5, t=5.888, \mathrm{df}=4,{ }^{*} p=0.0042$, paired $t$ test. ZX1: Baseline versus CPA (minutes 16-20): $n=4, t=0.9784, \mathrm{df}=3$, not significant, $p=0.4000$, paired $t$ test. BAPTA: Baseline versus CPA (minutes 16-20): $n=6, t=0.2515, \mathrm{df}=5$, not significant, $p=0.8114$, paired $t$ test. Figure $5 E$ : CPA potentiation (\%): Control: $21.92 \pm 3.006, n=5$. ZX1: $-3.017 \pm 5.401, n=4$. BAPTA: 0.1296 $\pm 5.53, n=6$. One-way ANOVA: $F=7.434,{ }^{*} p=0.0079$; Control versus ZX1: ${ }^{\star} p=0.0109$; Control versus BAPTA: ${ }^{\star} p=0.0133$; Bonferroni's multiple comparisons test. Figure 5F: Control: Baseline versus ZX1 (minutes 16-20): $n=5, t=3.116, \mathrm{df}=4,{ }^{*} p=0.0357$, paired $t$ test. CPA: Baseline versus ZX1 (minutes 16-20): $n=5, t=0.7067, \mathrm{df}=4$, not significant, $p=0.5187$, paired $t$ test. Figure 5G: ZX1 potentiation (\%): Control: $41.93 \pm 14.35, n=5$. CPA: $2.712 \pm 5.347, n=5$. Control versus CPA: $t=2.561, \mathrm{df}=8,{ }^{\star} p=0.0336$, unpaired $t$ test. Figure 5H: Baseline versus ZX1 (minutes 16-20): $n=5$, $t=7.558, \mathrm{df}=4,{ }^{*} p=0.0016$, paired $t$ test.

Figure 6A: Control: Baseline versus ZX1 (minutes 11-15): $n=5$, $t=3.398, \mathrm{df}=4,{ }^{*} p=0.0273$, paired $t$ test. HFS: Baseline versus ZX1 (minutes 11-15): $n=6, t=1.658, \mathrm{df}=5$, not significant, $p=0.1583$, paired $t$ test. HFS + LY367385/MPEP: Baseline versus ZX1 (minutes 11-15): $n=5, t=3.061, \mathrm{df}=4,{ }^{\star} p=0.0376$, paired $t$ test. Figure $6 B: \mathrm{ZX} 1$ potentiation (\%): Control: $43.5 \pm 9.4 \%, n=5$. HFS: $9.2 \pm 5.2 \%, n=6$. HFS + LY267385/MPEP: $42.6 \pm 8.2 \%, n=5$. One-way ANOVA: $F=$ $7.115,{ }^{\star} p=0.0082$. Control versus HFS: ${ }^{*} p=0.0123$; HFS versus HFS + LY367385, MPEP: ${ }^{\star} p=0.0148$; Bonferroni's multiple comparisons test. Figure 6C: EPSC (\% baseline): Control: $300 \mathrm{nM}: n=3,90.68 \pm 1.051 \%$; 1 $\mu \mathrm{M}: n=5,70.89 \pm 3.943 \%$; $3 \mu \mathrm{M}: n=5,54.61 \pm 2.791 \% ; 10 \mu \mathrm{M}: n=3$, $40.72 \pm 4.845 \%$. HFS: $300 \mathrm{nM}: n=3,90.24 \pm 4.327 \% ; 1 \mu \mathrm{M}: n=4$, $69.52 \pm 2.208 \% ; 3 \mu \mathrm{M}: n=4,52.1 \pm 3.214 \% ; 10 \mu \mathrm{M}: n=3,37.89 \pm$ $1.533 \%$. Nonlinear fits: Control: Hill slope $=1.095, R^{2}=0.9472$. HFS: Hill slope $=1.128, R^{2}=0.9765$. Figure $6 D: \operatorname{IC}_{50}(\mu \mathrm{M})$ : Control: $1.284 \pm 0.3566$. HFS: $1.267 \pm 0.2321$. Extra sum-of-squares $F$ test: not significant, $p=0.9687$. Figure $6 E$ : Control: Baseline versus after HFS (maximum $\Delta \mathrm{R} / \mathrm{R}$ ): $n=6, t=9.761$, df $=5,{ }^{\star} p=0.0002$, paired $t$ test. 
LY367385/MPEP: Baseline versus after HFS (maximum $\Delta \mathrm{R} / \mathrm{R}$ ): $n=6$, $t=4.013, \mathrm{df}=5,{ }^{*} p=0.0102$, paired $t$ test. Figure $6 F$ : Normalized zinc fluorescence (maximum $\Delta \mathrm{R} / \mathrm{R}$ ) (\% baseline): HFS: $44.07 \pm 2.554 \%, n=6$. HFS + LY367385/MPEP: $65.81 \pm 3.407 \%, n=6$. HFS versus HFS + LY367385/MPEP: $t=5.106, \mathrm{df}=10,{ }^{*} p=0.0005$, unpaired $t$ test.

Figure 7A: NE: Baseline versus ZX1 (minutes 21-25): $n=5, t=1.419$, $\mathrm{df}=4$, not significant, $p=0.2289$, paired $t$ test. NE + AIDA: $n=6$, $t=4.775$, df $=5,{ }^{*} p=0.005$, paired $t$ test. Figure $7 B$ : ZX1 potentiation (\%): NE: $11.7 \pm 8.56 \%, n=5$. NE + AIDA: $43.8 \pm 8.05 \%, n=6$. NE versus NE + AIDA: $t=2.724, \mathrm{df}=9,{ }^{*} p=0.024$, unpaired $t$ test. Figure 7C: paired-pulse ratio (PPR): NE: $1.954 \pm 0.16, n=6 . \mathrm{NE}+$ AIDA: $2.023 \pm 0.09, n=8$. NE versus NE + AIDA: $t=0.3901, \mathrm{df}=12$, not significant, $p=0.7033$, unpaired $t$ test; $1 /$ coefficient of variation $(C V)^{2}$ : NE: $49.35 \pm 7.77, n=6$. NE + AIDA: $43.42 \pm 9.06, n=8$. NE versus NE + AIDA: not significant, $p=0.4908$, Mann-Whitney test. Figure 7E: ABR threshold (dB SPL): Sham ipsilateral: $43.75 \pm 3.24, n=8$. NE contralateral: $68.33 \pm 3.07, n=6$. NE + AIDA contralateral: 65.71 $\pm 2.97, n=7$. Kruskal-Wallis test: ${ }^{*} p=0.0002$. Sham ipsilateral versus NE contralateral: ${ }^{*} p=0.0042$; Sham ipsilateral versus NE + AIDA contralateral: ${ }^{*} p=0.0076$; NE contralateral versus NE + AIDA contralateral: not significant, $p>0.9999$; Dunn's multiple comparisons test. ABR Wave I $(\mu \mathrm{V})$ : Sham ipsilateral: $2.67 \pm 0.31, n=8$. NE contralateral: $1.23 \pm 0.13$, $n=6$. NE + AIDA contralateral: $1.25 \pm 0.27, n=7$. Kruskal-Wallis test: ${ }^{\star} p=0.0024$. Sham ipsilateral versus NE contralateral: ${ }^{*} p=0.0387$; Sham ipsilateral versus NE + AIDA contralateral: ${ }^{\star} p=0.0107$; NE contralateral versus NE + AIDA contralateral: not significant, $p>0.9999$; Dunn's multiple comparisons test.

\section{Results}

Bidirectional activity-dependent long-term synaptic zinc plasticity requires $\mathrm{G} 1 \mathrm{mGluR}$ activation

To investigate the mechanisms underlying synaptic zinc plasticity, we first determined whether we could induce long-term synaptic zinc plasticity in DCN PF synapses in mouse brain slices. In these synapses, synaptic zinc inhibits AMPAR and NMDAR EPSCs via postsynaptic mechanisms. Zinc inhibition is evidenced by using ZX1, a fast, high-affinity extracellular zinc chelator, which potentiates AMPAR and NMDAR EPSCs (Anderson et al., 2015; Kalappa et al., 2015; Kalappa and Tzounopoulos, 2017). Under the conditions we used here, the ZX1 potentiation of AMPAR and NMDAR EPSCs is dependent on synaptic stimulation and ZnT3 (Anderson et al., 2015; Kalappa et al., 2015; Kalappa and Tzounopoulos, 2017). ZnT3 is the transporter that loads zinc into synaptic vesicles (Palmiter et al., 1996; Cole et al., 1999). Therefore, the zinc inhibition of AMPAR and NMDAR EPSCs is mediated by ZnT3-dependent, synaptically released zinc, but not tonic zinc (Anderson et al., 2015; Kalappa et al., 2015). In this study, we used the amount of ZX1 potentiation of AMPAR and NMDAR EPSCs to monitor synaptic zinc signaling, and long-term synaptic zinc plasticity in DCN PF synapses.

Consistent with previous studies, we found that ZX1 potentiated postsynaptic PF AMPAR EPSCs in DCN CWCs, a class of inhibitory interneurons (Fig. 1A,B) (Kalappa et al., 2015; Kalappa and Tzounopoulos, 2017). We then tested whether we could induce long-term synaptic zinc plasticity by using patterns of synaptic activation that induce long-term plasticity of glutamatergic synaptic strength in DCN PF synapses, such as LTP and LTD (Fujino and Oertel, 2003; Tzounopoulos et al., 2004, 2007). We started by examining the effect of ZX1 on AMPAR EPSCs following HFS of PFs (HFS, $3 \times 100 \mathrm{~Hz}$ for $1 \mathrm{~s}, 10 \mathrm{~s}$ interstimulus interval), which induces LTP (Fujino and Oertel, 2003). After obtaining a stable response following LTP induction (Fig. $1 C$ ), we renormalized AMPAR EPSC amplitude to quantify the amount of ZX1 potentiation (Fig. 1C). After HFS, ZX1 application did not potentiate AMPAR EPSCs (Fig. 1C, blue), whereas
ZX1 potentiated AMPAR EPSCs in control experiments where HFS was not applied (Fig. 1C, red, Fig. 1D). The loss of ZX1 potentiation indicates a loss of zinc-mediated inhibition of AMPARs, suggesting that HFS caused a long-term reduction in synaptic zinc signaling, termed Z-LTD (Fig. 1D). To confirm that the HFS-induced reduction of ZX1 potentiation is not due to a gradual decline in EPSC amplitude after HFS, we performed an additional control experiment where we delivered HFS but did not apply ZX1 (Fig. 1E). AMPAR EPSC amplitude remained elevated and stable following HFS, over the duration of our recordings (Fig. 1E). This result supports that the reduced ZX1 potentiation after HFS (Fig. 1C, blue) cannot be explained by a gradual decline in EPSC amplitude after HFS, further supporting our conclusion that ZX1 does not potentiate AMPAR EPSCs after HFS, due to a reduction in zinc signaling.

To quantify the average effect of ZX1 after HFS, we renormalized EPSCs to the baseline before ZX1 application, because not every cell reached a new stable baseline at the same time following HFS ( $\sim 20-25 \mathrm{~min}$ after HFS); thus, ZX1 was not always applied at the exact same time. However, in Figure $1 F$, we show three example cells without renormalization after HFS, in which ZX1 was applied at the exact same time. These data show that ZX1 potentiates EPSCs in a control cell where no HFS was applied (Fig. $1 F$, red) but does not potentiate EPSCs in a cell that received HFS (Fig. 1F, blue; ZX1 was not applied in gray). Together, these results suggest that Z-LTD can be induced in DCN PF synapses, as evidenced by reduced zinc-mediated inhibition of AMPAR EPSCs.

In Figure $1 C, D$, and in general throughout this study, we used HFS to induce activity-dependent reductions of synaptic zinc signaling (Z-LTD). The elimination of ZX1 potentiation after HFS (Fig. $1 C, D$ ) suggests that HFS occluded the ZX1 potentiation by reducing zinc signaling. However, HFS also induces LTP via glutamate-based effects. Our conclusion that HFS causes an activity-dependent reduction of synaptic zinc signaling, and the delineation of zinc- versus glutamate-based effects on synaptic plasticity are further explored and supported by electrophysiological and imaging approaches throughout this study (see Figs. 2-6).

After establishing that HFS caused Z-LTD, we then studied the underlying mechanisms. NMDARs contribute to the induction of LTP and LTD in the DCN and most central synapses (Malenka and Nicoll, 1993; Fujino and Oertel, 2003; Tzounopoulos et al., 2004, 2007). To test the role of NMDARs in the induction of Z-LTD, we blocked NMDARs with APV (NMDAR antagonist, 50 $\mu \mathrm{M}$ ). Similar to the lack of ZX1 potentiation after HFS (Fig. $2 A$, blue), ZX1 did not potentiate AMPAR EPSCs after HFS in the presence of APV (Fig. 2A, pink), indicating that NMDARs are not required for the induction of Z-LTD.

Under these conditions, HFS induced NMDAR-independent LTP and Z-LTD (Fig. 2A). Based on this finding, we hypothesized that HFS-induced Z-LTD might contribute to the HFSinduced LTP. To delineate zinc- versus glutamate-based effects on synaptic plasticity, we first applied ZX1 before HFS, thus removing the zinc-based effects of HFS. We found that HFS did not induce LTP under these conditions, suggesting that HFSinduced Z-LTD contributes to HFS-induced NMDAR-independent LTP (Fig. 2B).

$\mathrm{PF}$ synapses in the DCN also exhibit glutamatergic plasticity that involves metabotropic glutamate receptor (mGluR) signaling (Fujino and Oertel, 2003). Because G1 mGluRs are expressed in CWCs and in the DCN molecular layer, where PF terminals and CWCs reside (Wright et al., 1996; Bilak and Morest, 1998), we next 
tested whether G1 mGluR activation is necessary for Z-LTD induction. To test this hypothesis, we repeated the experiment shown in Figure $2 A$, but we now blocked G1 mGluRs with LY367385 (100 $\mu \mathrm{M}$, mGluR1-selective antagonist) and MPEP ( $4 \mu \mathrm{M}$, mGluR5-selective antagonist). Under these conditions, ZX1 potentiation was observed after HFS (Fig. 2C, $D$, green), indicating that LY367385/MPEP blocked HFS-induced Z-LTD. This ZX1 potentiation was not different compared with control experiments where APV and LY367385/MPEP were applied but HFS was not delivered (Fig. $2 C, D$, red, and Fig. $2 D$, green vs red). Together, these results demonstrate that blockade of G1 mGluRs eliminated HFS-induced Z-LTD; therefore, G1 mGluR activation is necessary for Z-LTD induction.

Although G1 mGluR antagonists blocked Z-LTD, LTP was induced under these conditions (Fig. 2C, green). Moreover, the magnitude of this LTP was not different from the LTP induced in the absence of G1 mGluR antagonists (LTP in Fig. $2 A$ vs 2 Fig. $2 C$; $p=0.886$, unpaired $t$ test). This is unexpected, as we would expect to observe larger LTP magnitude in the absence of G1 mGluR antagonists (Fig. 2A), because the concurrent induction of Z-LTD would further enhance AMPAR EPSC amplitude due to reduced zinc inhibition. Conversely, we would expect to observe less LTP in the presence of G1 mGluR antagonists (Fig. 2C), where Z-LTD is blocked; thus, AMPAR EPSCs would be more inhibited by zinc. These results demonstrate a separation between zinc- versus glutamate-based effects on synaptic plasticity. Namely, these results suggest that a G1 mGluR-independent glutamate-based mechanism mediates LTP when Z-LTD is blocked.

Glutamatergic plasticity is bidirectional: synapses undergo LTP or LTD in response to HFS or LFS, respectively (Mulkey and Malenka, 1992; Malenka and Nicoll, 1993; Fujino and Oertel, 2003). To determine whether long-term synaptic zinc plasticity is bidirectional, we tested whether LFS increases zinc signaling. Because the induction of Z-LTD is mGluR-dependent (Fig. $2 C, D)$, we used an LFS stimulation protocol previously shown to favor mGluR-dependent synaptic plasticity (Oliet et al., 1997).

Namely, we used LFS ( $5 \mathrm{~Hz}, 3 \mathrm{~min}$ ), APV to block NMDARs, and high extracellular concentrations of divalent ions $(4 \mathrm{mM}$ $\mathrm{Ca}^{2+}$ and $\mathrm{Mg}^{2+}$ ) (Oliet et al., 1997). Compared with interleaved control experiments where no LFS was applied (Fig. 3A, red), LFS increased the amount of subsequent ZX1 potentiation (Fig. $3 A$, cyan, and Fig. $3 D$, cyan vs red). Increased ZX1 potentiation indicates increased zinc-mediated inhibition of AMPARs, suggesting that LFS caused a long-term increase in synaptic zinc signaling, termed Z-LTP (Fig. 3D). Control ZX1 potentiation in these conditions (Fig. $3 A, D$, red) was slightly less, albeit not significantly different $(p=0.11$, unpaired $t$ test $)$ than previous
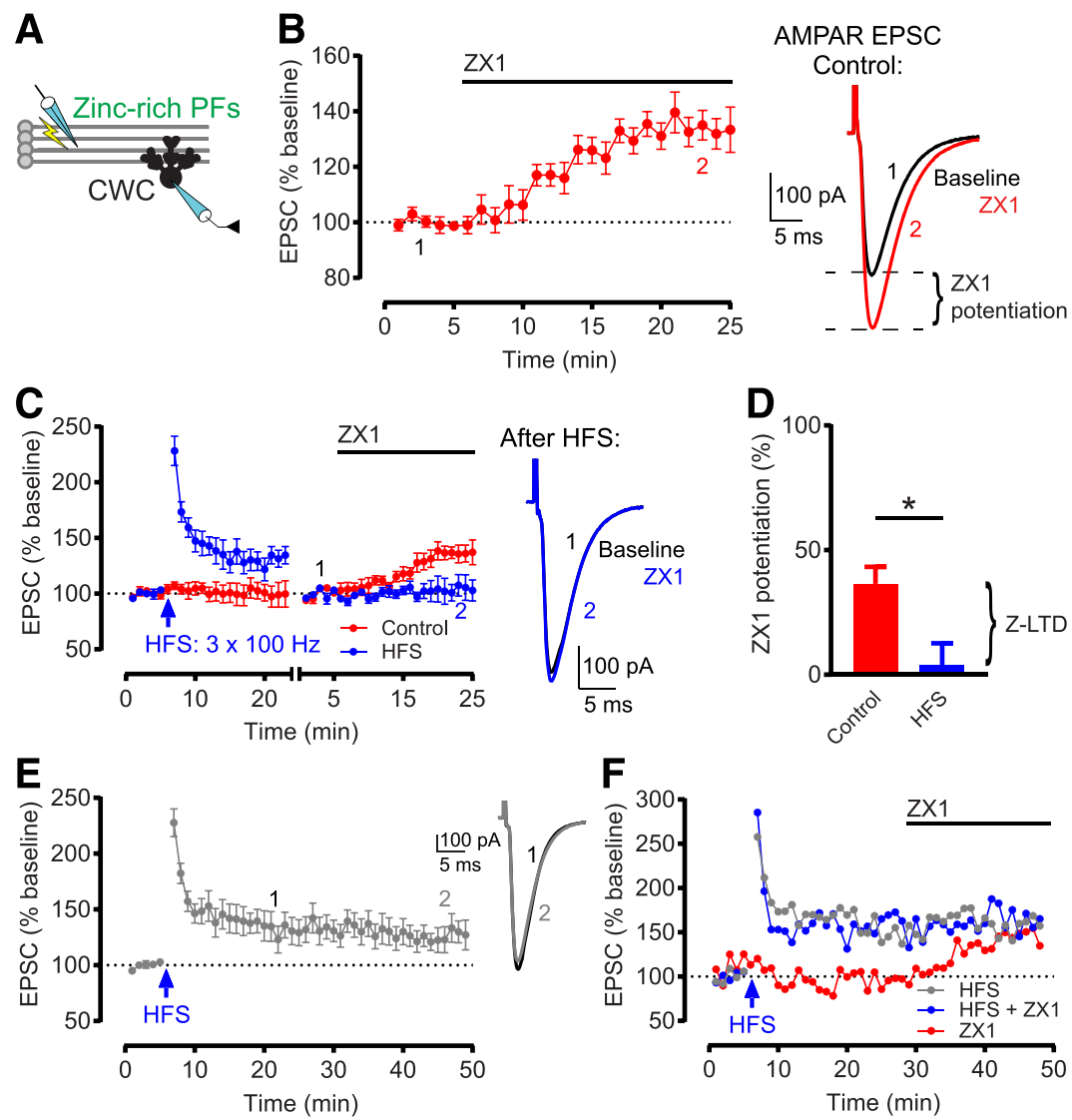

Figure 1. HFS induces Z-LTD in DCN PF synapses. $A$, Schematic of experimental setup illustrating stimulation of zincrich glutamatergic DCN PFs and whole-cell recording of a postsynaptic CWC. $\boldsymbol{B}$, Left, Time course of AMPAR EPSC amplitude before and after ZX1 application, normalized to baseline before ZX1 application (100 $\mu \mathrm{M})$. ZX1 significantly potentiated AMPAR EPSCs ( $n=10,{ }^{*} p=002$, Wilcoxon matched-pairs signed rank test). Right, Example AMPAR EPSCs before and after ZX1 application, showing ZX1 potentiation. C, Left, Time course of AMPAR EPSC amplitude before and after HFS, and before and after subsequent ZX1 application (blue), and similar time course in control experiments (without HFS, red). After obtaining a stable baseline, HFS was delivered $(3 \times 100 \mathrm{~Hz}$ for $1 \mathrm{~s}, 10 \mathrm{~s}$ interstimulus interval). HFS induced LTP ( $n=7,{ }^{*} p=0.02$, paired $t$ test). To examine ZX1 potentiation after HFS, after obtaining a stable baseline tion is indicated by a gap and restart of timing in the $x$ axis. In control experiments (red), ZX1 potentiated EPSCs ( $n=6,{ }^{*} p=0.03$, Wilcoxon matched-pairs signed rank test). After HFS (blue), ZX1 did not potentiate EPSCs ( $n=7$, Average ZX1 potentiation (\% increase from baseline) during the last 5 min of ZX1 application (minutes 21-25). Control $(n=6)$ versus HFS $(n=7):{ }^{*} p=0.016$, unpaired $t$ test. The reduction in ZX1 potentiation is termed Z-LTD. $\boldsymbol{E}$, Time course of AMPAR EPSC amplitude before and after HFS, without subsequent ZX1 application. HFS induced LTP ( $n=8,{ }^{*} p=0.027$, paired $t$ test). After HFS, EPSCs remained stable for the duration of the recording (minutes vs minutes 45-49: $n=5$, not significant, $p=0.12$, paired $t$ test). Example traces represent AMPAR EPSCS from minutes $19-23$ and minutes $45-49$. $\boldsymbol{F}$, Example time courses from 3 individual cells, without renormalization, where ZX1 was applied at the exact same time. Blue represents that HFS was delivered and ZX1 was subsequently but $Z X 1$ was not applied. For all figures, data are mean \pm SEM. ${ }^{*} p<0.05$. For detailed values and statistical tests for all figures, see Statistical analysis.

control experiments performed in ACSF with $2.4 / 1.3 \mathrm{mM}$ of extracellular $\mathrm{Ca}^{2+} / \mathrm{Mg}^{2+}$ (Fig. $1 B$ ). This is likely due to reduced neuronal excitability in higher divalent concentrations (Oliet et al., 1997; Kalappa et al., 2015). Together, these results show that LFS induced Z-LTP, thus demonstrating that activity-dependent plasticity of zinc signaling is bidirectional: HFS induces LTD of zinc signaling (Z-LTD), whereas LFS induces LTP of zinc signaling (Z-LTP).

Although LFS induced Z-LTP, which increases zinc-mediated inhibition of AMPAR EPSCs, LFS did not induce LTD (Fig. 3A, cyan). Based on this finding, we hypothesized that LFS also induces a concurrent LTP that might compensate for the depressant 

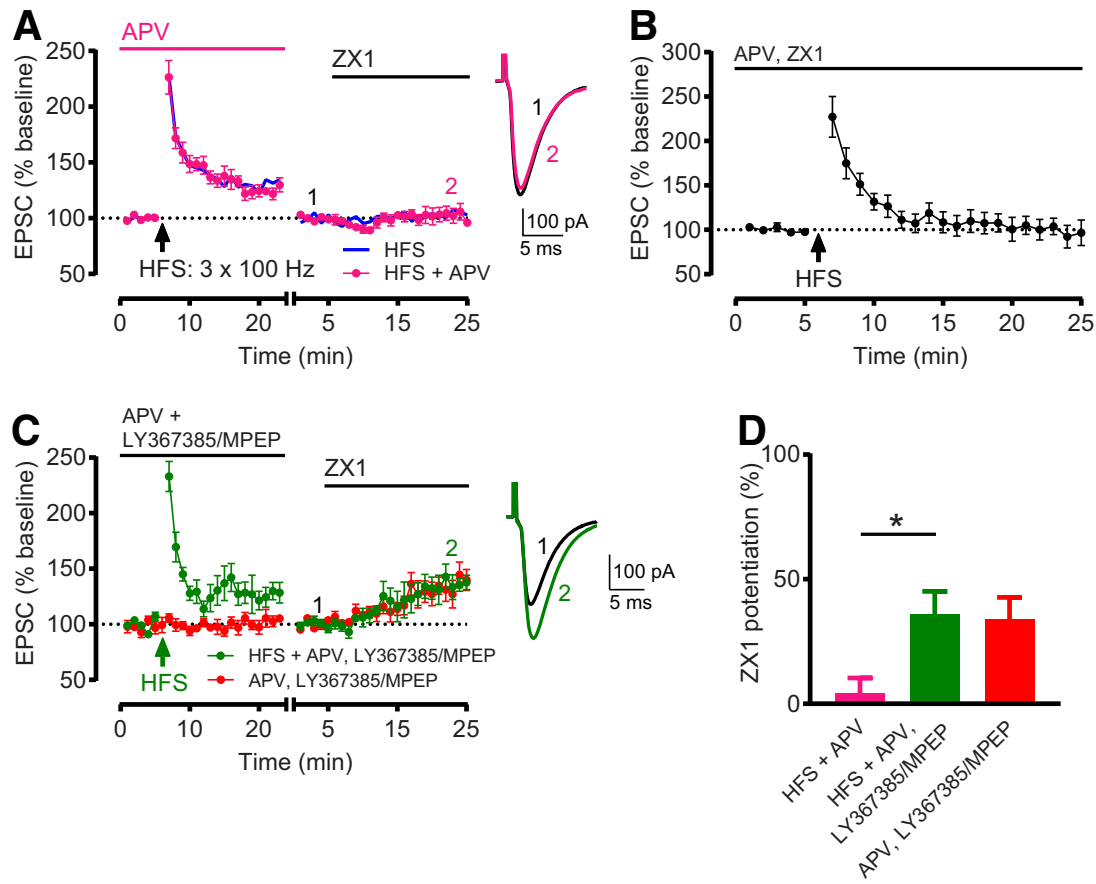

Figure 2. G1 mGluR activation is required for HFS-induced Z-LTD. A, Time course of AMPAR EPSC amplitude before and after HFS in the presence of APV $(50 \mu \mathrm{M})$, and before and after subsequent ZX1 application (pink). HFS induced LTP $(n=9$, ${ }^{*} p=0.001$, paired $t$ test). After HFS + APV, ZX1 did not potentiate EPSCS $(n=9$, not significant, $p=0.82$, Wilcoxon matchedpairs signed rank test). Blue line indicates HFS time course replotted from Figure 1C. The presence of APV during HFS did not affect the induction of Z-LTD. ZX1 potentiation (\%) HFS versus HFS + APV: not significant, $p=0.96$, unpaired $t$ test. $\boldsymbol{B}$, Time course of AMPAR EPSC amplitude before and after HFS in the presence of APV $(50 \mu \mathrm{M})$ and ZX1 (100 $\mu \mathrm{M})$. HFS did not induce LTP ( $n=6$, not significant, $p=0.699$, paired $t$ test). C, Similar time course as in $A$, but with HFS in the presence of LY367385 $(100 \mu \mathrm{M})$, MPEP $(4 \mu \mathrm{M})$, and APV $(50 \mu \mathrm{M})$ (green), compared with cells with LY367385, MPEP, and APV but without HFS (red). HFS induced LTP ( $n=6,{ }^{*} p=0.029$, paired $t$ test). After HFS + APV, LY367385/MPEP, ZX1 potentiated EPSCS $\left(n=5,{ }^{*} p=0.02\right.$, paired $t$ test). $A, C$, To examine $Z X 1$ potentiation after HFS, similar approach and renormalization as in Figure $1 C$ were performed. Example traces represent AMPAR EPSCs before and after ZX1. D, Average ZX1 potentiation (\% increase from baseline) during the last 5 min of ZX1 application (minutes 21-25). HFS + APV, LY367385/MPEP $(n=5)$ increased ZX1 potentiation compared with HFS + APV $(n=9)\left({ }^{*} p=0.0181\right)$, and was not different from APV, LY367385/MPEP $(n=5)$ (not significant, $\left.p>0.99\right)$. Oneway ANOVA/Bonferroni.

effect of Z-LTP. To separate these effects, we applied ZX1 before LFS. We found that LFS caused a significant LTP (Fig. 3B). This result suggests that LFS also induces an LTP that compensates for the depressant effect of Z-LTP.

We next tested whether G1 mGluR activation is necessary for the induction of Z-LTP. After delivering LFS in the presence of LY367385/MPEP, ZX1 potentiation was reduced compared with LFS alone (Fig. $3 C$ green, and Fig. 3D, cyan vs green), and was similar to ZX1 potentiation in control experiments where LY367385/MPEP were applied but LFS was not delivered (Fig. $3 C$, gray, and Fig. $3 D$, green vs gray). This result indicates that LY367385/MPEP blocked Z-LTP. To confirm that the changes in ZX1 potentiation after LFS (or LFS with LY367385/MPEP) are not due to gradual changes in EPSC amplitude after LFS, we performed additional control experiments where we delivered either LFS alone (Fig. 3E, cyan) or LFS with LY367385/MPEP (Fig. 3E, green) but did not apply ZX1. In these experiments, AMPAR EPSC amplitude remained stable following LFS, further supporting our conclusion that the observed changes in ZX1 potentiation after LFS are due to changes in zinc signaling. These results indicate that G1 mGluR activation is necessary for the induction of Z-LTP. Together, these results reveal that activation of G1 mGluR signaling is necessary for the induction of both Z-LTP and Z-LTD.
G1 mGluR activation is sufficient to induce bidirectional long-term synaptic zinc plasticity Is activation of $\mathrm{G} 1 \mathrm{mGluRs}$ sufficient to induce Z-LTP and Z-LTD? Because G1 mGluRs are required for both increases and decreases in synaptic zinc signaling by different stimulation paradigms, we hypothesized that the direction of plasticity depends on the differential activation of G1 mGluRs during HFS and LFS. To test this, we applied high or low concentrations of DHPG (G1 mGluR agonist, 50 or 5 $\mu \mathrm{M})$. Consistent with previous studies (Huber et al., 2001; Snyder et al., 2001; Wiśniewski and Car, 2002), application of $50 \mu \mathrm{M}$ DHPG caused a significant depression of synaptic strength (Fig. $4 A$, cyan). After applying $50 \mu \mathrm{M}$ DHPG, obtaining a new stable baseline, and then applying ZX1, we observed that the ZX1 potentiation of EPSCs was significantly increased (Fig. $4 A$, cyan) compared with control experiments where DHPG was not applied (Fig. 4A, red, and Fig. 4B, cyan vs red). This result indicates that 50 $\mu \mathrm{M}$ DHPG increases synaptic zinc signaling: G1 mGluR activation is sufficient to induce Z-LTP.

We next tested whether application of a lower concentration of DHPG causes Z-LTD. After applying $5 \mu \mathrm{M}$ DHPG and obtaining a new stable baseline, ZX1 did not potentiate EPSCs (Fig. $4 A$, purple, and Fig. $4 B$, purple vs red), consistent with Z-LTD induction. To confirm that the changes in ZX1 potentiation after 50 or $5 \mu \mathrm{M}$ DHPG are not due to changes in EPSC amplitude after DHPG (in the absence of ZX1), we performed additional control experiments where we applied 50 or $5 \mu \mathrm{M}$ DHPG but did not apply ZX1. We found that, after application of $50 \mu \mathrm{M}$ DHPG, EPSCs remained depressed and stable for the duration of the recording (Fig. 4C, cyan); and after application of $5 \mu \mathrm{M}$ DHPG, EPSCs remained stable for the duration of the recording (Fig. $4 C$, purple). These results further support our conclusions that enhanced ZX1 potentiation after 50 $\mu \mathrm{M}$ DHPG is due to increased zinc signaling, whereas decreased ZX1 potentiation after $5 \mu \mathrm{M}$ DHPG is due to decreased zinc signaling. Together, these results demonstrate that G1 mGluR activation is sufficient to cause bidirectional synaptic zinc plasticity. Furthermore, the direction of zinc plasticity depends on the concentration of DHPG: $50 \mu \mathrm{M}$ DHPG causes Z-LTP, whereas $5 \mu \mathrm{M}$ DHPG causes Z-LTD (Fig. 4B). These results are consistent with the notion that bidirectional zinc plasticity depends on differential activation of G1 mGluRs by either LFS/HFS or high/low concentrations of DHPG.

Because application of $50 \mu \mathrm{M}$ DHPG induced Z-LTP and synaptic depression (Fig. 4A, cyan), we hypothesized that Z-LTP might contribute to the synaptic depression. To test this hypothesis, we applied ZX1 before $50 \mu \mathrm{M}$ DHPG. We found that 50 $\mu \mathrm{M}$ DHPG did not cause significant synaptic depression (Fig. 
A
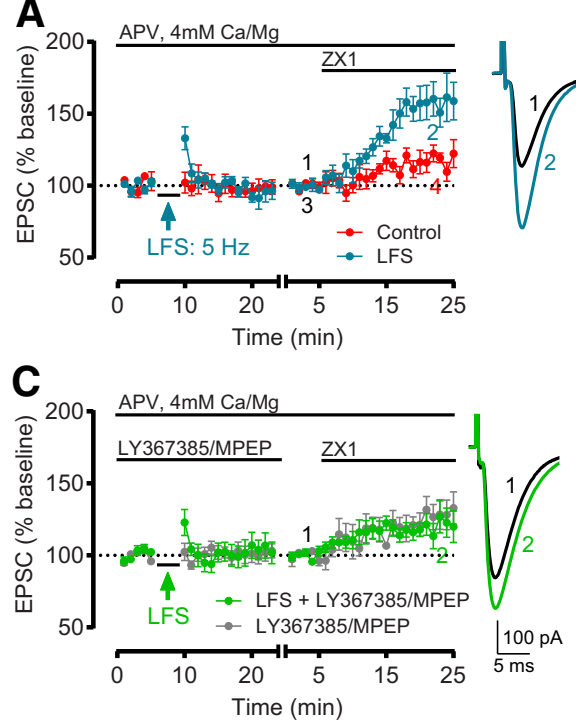
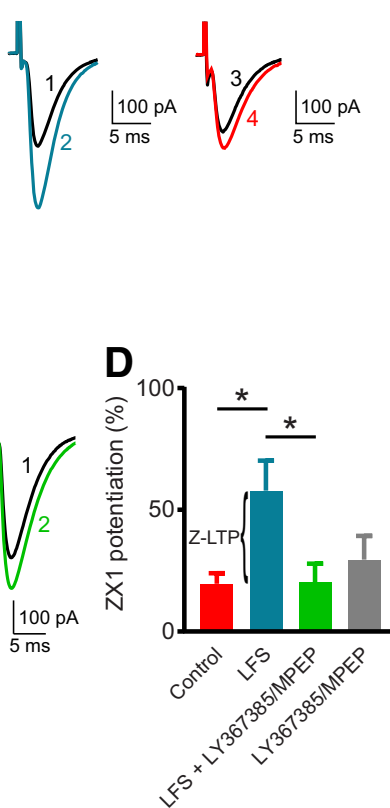

B
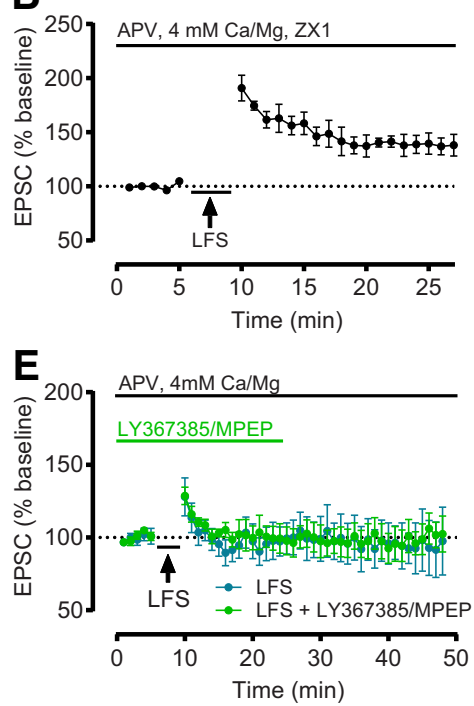

Figure 3. LFS induces Z-LTP, which requires $\mathrm{G} 1 \mathrm{mGluR}$ activation. $\boldsymbol{A}$, Time course of AMPAR EPSC amplitude before and after LFS (5 Hz, $3 \mathrm{~min})$, and before and after subsequent ZX1 application (cyan); and similar time course in interleaved control experiments (without LFS, red). LFS did not induce LTD ( $n=8$, not significant, $p=0.22$, paired $t$ test). ZX1 potentiated EPSCs after LFS $\left(n=6,{ }^{*} p=0.0025\right.$, paired $t$ test) and in controls $\left(n=5,{ }^{*} p=0.019\right.$, paired $t$ test). $\boldsymbol{B}$, Time course of AMPAR EPSC amplitude before and after LFS, in the presence of ZX1 (100 $\mu$ M). LFS induced LTP ( $n=5,{ }^{*} p=0.027$, paired $t$ test). C, Similar time course as in A, but with LFS in the presence of LY367385 (100 $\mu$ M) and MPEP (4 $\mu$ M) (green), compared with cells with LY367385 and MPEP but without LFS (gray). LFS + LY367385/MPEP did not induce LTD ( $n=6$, not significant, $p=0.69$, paired $t$ test). ZX1 potentiated EPSCS after LFS ( $n=6$, * $p=0.02$, paired $t$ test) and without LFS ( $n=6,{ }^{*} p=0.015$, paired $t$ test). $\boldsymbol{A}, \boldsymbol{C}$, To examine the ZX1 potentiation after LFS, similar approach and renormalization as in Figure $1 C$ were performed. Example traces represent AMPAR EPSCs before and after ZX1. $\boldsymbol{D}$, Average ZX1 potentiation (\% increase from baseline) during the last 5 min of ZX1 application (minutes $21-25)$. Control: $n=5$; LFS: $n=6$; LFS + LY367385/MPEP: $n=6$; LY367385/MPEP: $n=6$. LFS increased ZX1 potentiation compared with control ( $\left.{ }^{*} p=0.03\right)$; this increase was blocked by LY367385 and MPEP $\left({ }^{*} p=0.027\right)$; and LFS + LY367385/MPEP was not different from LY367385/MPEP alone (not significant, $\left.p>0.99\right)$. One-way ANOVA/Bonferroni. The increase in ZX1 potentiation is termed ZLTP. $E$, Time course of AMPAR EPSC amplitude before and after LFS, with and without LY367385/MPEP. After LFS, EPSCs remained stable for the duration of the recording. LFS: $n=4$; minutes 19-23 versus minutes 44-48: not significant, $p=0.32$, paired $t$ test. LFS + LY367385/MPEP: $n=4$, minutes 19-23 versus minutes 44-48: not significant, $p=0.87$, Wilcoxon matched-pairs signed rank test.

$4 D$ ), suggesting that the increased zinc signaling (Z-LTP) contributes to the synaptic depression caused by $50 \mu \mathrm{M}$ DHPG.

Although application of $5 \mu \mathrm{M}$ DHPG induced Z-LTD, which reduces zinc inhibition of AMPAR EPSCs, it did not cause LTP (Fig. $4 A$, purple). Therefore, we hypothesized that $5 \mu \mathrm{M}$ DHPG also induced a concurrent synaptic depression that might compensate for the enhancing effect of Z-LTD. To separate these effects, we applied ZX1 before $5 \mu \mathrm{M}$ DHPG. We found that 5 $\mu \mathrm{M}$ DHPG caused a modest, albeit significant, synaptic depression under these conditions (Fig. $4 E$ ). This result suggests that 5 $\mu \mathrm{M}$ DHPG does not increase AMPAR EPSC amplitude because it also induces a depression that compensates for the enhancing effect of Z-LTD.

Electrical synaptic stimulation with LFS/HFS or pharmacological activation of G1 mGluRs with high/low concentrations of DHPG induce bidirectional synaptic zinc plasticity; however, it is unknown whether these two different methods induce mechanistically similar synaptic zinc plasticity. Because we cannot address this hypothesis using Z-LTD, as both HFS and $5 \mu \mathrm{M}$ DHPG eliminate ZX1 potentiation, we compared the amount of Z-LTP elicited by applying sequential LFS and $50 \mu \mathrm{M}$ DHPG to the amount of Z-LTP elicited by LFS or $50 \mu \mathrm{M}$ DHPG alone. If electrical and pharmacological manipulations induce Z-LTP by different mechanisms, then LFS and $50 \mu \mathrm{M}$ DHPG application should yield an additive effect on Z-LTP, and subsequent ZX1 potentiation should be greater than that following LFS alone or application of $50 \mu \mathrm{M}$ DHPG alone. To test this, we performed interleaved experiments to determine the effect of $50 \mu \mathrm{M}$ DHPG alone (Fig. 4F) with experiments involving stimulation with LFS and subsequent DHPG application (Fig. 4G). We found that ZX1 potentiation after sequential LFS and $50 \mu \mathrm{M}$ DHPG was not different from ZX1 potentiation after either LFS or DHPG alone (Fig. 4H). Together, these results show that LFS occluded the effect of $50 \mu \mathrm{M}$ DHPG; thus, LFS and DHPG induce Z-LTP likely via a common mechanistic pathway.

\section{Rises in postsynaptic calcium are necessary and sufficient for inducing Z-LTD}

Because G1 mGluR activation and synaptic plasticity are often linked to rises in intracellular $\mathrm{Ca}^{2+}$ (Abdul-Ghani et al., 1996; Conn and Pin, 1997; Malenka and Bear, 2004; Kim et al., 2008), we tested whether rises in intracellular $\mathrm{Ca}^{2+}$ are necessary for $\mathrm{Z}$ LTD. To do this, we applied HFS while preventing rises in postsynaptic $\mathrm{Ca}^{2+}$ with intracellular BAPTA $(10 \mathrm{mM}$, in the pipette). After HFS in the presence of intracellular BAPTA, ZX1 potentiated AMPAR EPSCs (Fig. 5A). Moreover, the amount of ZX1 potentiation after HFS in the presence of BAPTA was not significantly different from the amount of ZX1 potentiation in BAPTA but without HFS (Fig. $5 B$, and Fig. $5 C$, orange vs black), suggesting that intracellular BAPTA blocked Z-LTD. Additionally, the amount of ZX1 potentiation in the presence of intracellular BAPTA was not different compared with ZX1 potentiation in the control (Fig. $5 B$ vs Fig. $1 B ; p=0.87$, unpaired $t$ test), indicating that BAPTA does not influence ZX1 potentiation under 

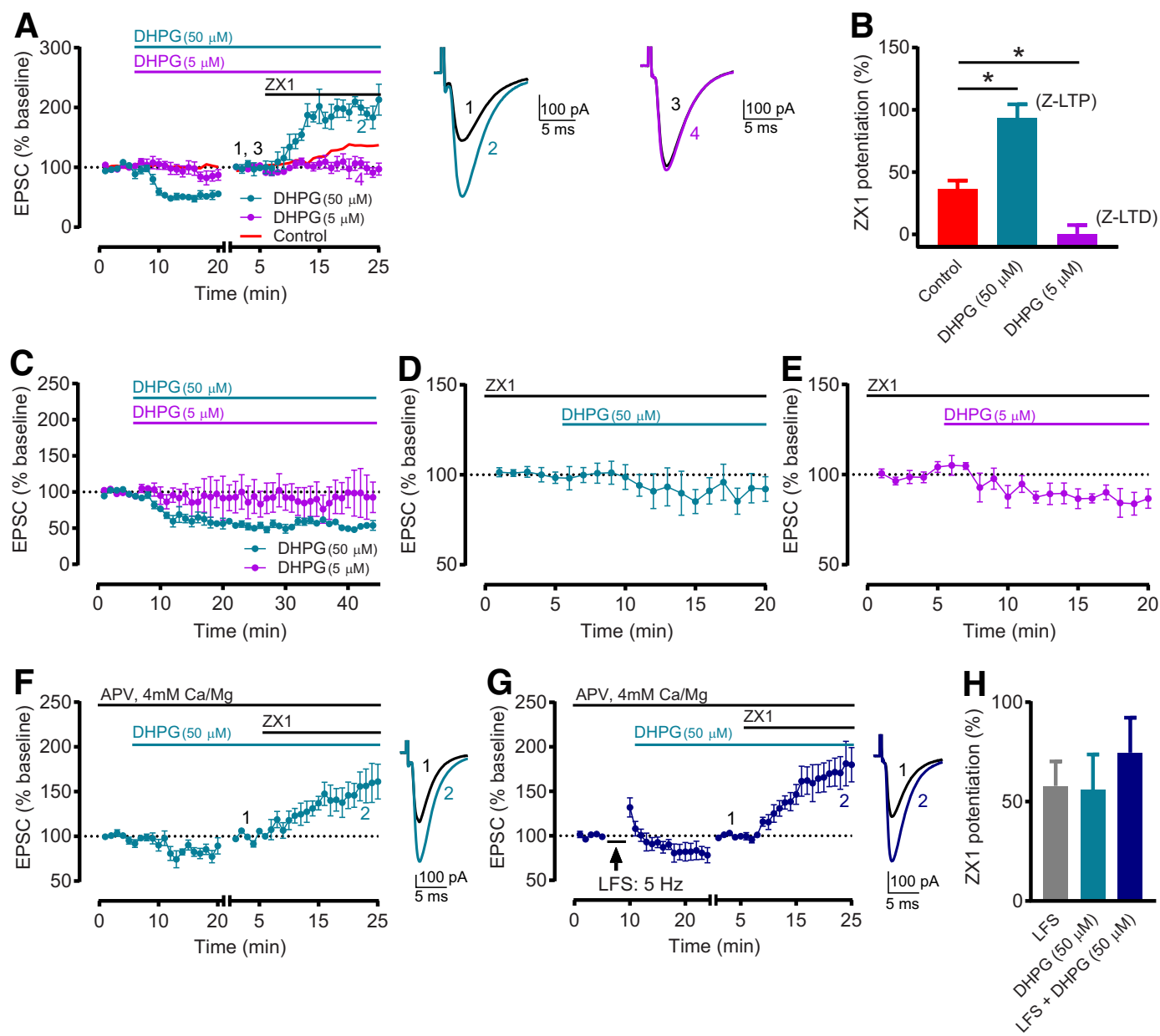

Figure 4. G1 mGluR activation is sufficient to induce Z-LTP and Z-LTD. A, Time course of AMPAR EPSC amplitude before and after application of $50 \mu \mathrm{M}$ DHPG (cyan) or $5 \mu M$ DHPG (purple), and before and after subsequent ZX1 application. Red line indicates Control time course replotted from Figure $1 C$. The $50 \mu \mathrm{M}$ DHPG caused synaptic depression $\left(n=6,{ }^{*} p=0.003\right.$, paired $t$ test), and ZX1 potentiated EPSCS $\left(n=5,{ }^{*} p=0.02\right.$, paired $t$ test). DHPG $(5 \mu \mathrm{M})$ did not change EPSC amplitude $(n=6$, not significant, $p=0.14$, paired $t$ test), and ZX1 did not potentiate EPSCS ( $n=5$, not significant, $p=0.81$, paired $t$ test). To examine the ZX1 potentiation after DHPG application, after obtaining a stable baseline after DHPG, AMPAR EPSC amplitude was renormalized to the new baseline before ZX1 application. The renormalization is indicated by a gap and restart of timing in the $x$ axis. Example traces represent AMPAR EPSCS before and after ZX1. $B$, Average ZX1 potentiation (\% increase from baseline) during the last 5 min of ZX1 application (minutes 21-25), compared with Control from Figure $1 C, D$. DHPG (50 $\mu$ M): $n=5$; DHPG (5 $\mu \mathrm{M}): n=5$. DHPG $(50 \mu \mathrm{M})$ increased ZX1 potentiation compared with control $\left({ }^{*} p=0.0006\right)$, whereas DHPG $(5 \mu \mathrm{M})$ reduced ZX1 potentiation compared with control ( $\left.{ }^{*} p=0.029\right)$. 0ne-way ANOVA/Bonferroni. Increased and decreased ZX1 potentiation correspond to Z-LTP and Z-LTD, respectively. C, Time course of AMPAR EPSC amplitude before and after application of $50 \mu M$ DHPG (cyan) or $5 \mu \mathrm{M}$ DHPG (purple). After $50 \mu \mathrm{M}$ DHPG, EPSCs remained depressed and stable for the duration of the recording (minutes $16-20$ vs minutes 40-44: $n=5$, not significant, $p=0.21$, paired $t$ test). After $5 \mu \mathrm{M}$ DHPG, EPSCs remained stable for the duration of the recording (minutes 16-20 vs minutes 40-44: $n=4$, not significant, $p=0.35$, paired $t$ test). $\boldsymbol{D}$, Time course of AMPAR EPSC amplitude before and after application of $50 \mu \mathrm{M}$ DHPG, in the presence of ZX1 (100 $\mu \mathrm{M})$. DHPG $(50 \mu \mathrm{M})$ did not cause synaptic depression ( $n=5$, not significant, $p=0.29$, paired $t$ test). $\boldsymbol{E}$, Similar time course as in $\boldsymbol{D}$, with $5 \mu \mathrm{M}$ DHPG. DHPG $(5 \mu \mathrm{M})$ caused synaptic depression $\left(n=5,{ }^{*} p=0.025\right.$, paired $t$ test). $\boldsymbol{F}$, Similar time course as in $\boldsymbol{A}$ (cyan), but in same extracellular conditions as in Figure $3 A, C$. DHPG $(50 \mu \mathrm{M})$ caused synaptic depression $\left(n=5,{ }^{*} p=0.04\right.$, paired $t$ test). ZX1 potentiated EPSCs $\left(n=5,{ }^{*} p=0.026\right.$, paired $t$ test). G, Time course of AMPAR EPSC amplitude before and after sequential LFS ( $5 \mathrm{~Hz}, 3 \mathrm{~min})$ and application of $50 \mu \mathrm{M}$ DHPG, and before and after subsequent ZX1 application, in same conditions as in $\boldsymbol{F}$. ZX1 potentiated EPSCS $\left(n=5,{ }^{*} p=0.004\right.$, paired $t$ test). $\boldsymbol{F}, \boldsymbol{G}$, to examine the ZX1 potentiation, similar approach and renormalization as in $\boldsymbol{A}$ was performed. Example traces represent AMPAR EPSCS before and after ZX1. $\boldsymbol{H}$, Average ZX1 potentiation (\% increase from baseline) during the last 5 min of ZX1 application (minutes 21-25) for the experiments in $\boldsymbol{F}, \boldsymbol{G}$, with LFS data from Figure 3D. DHPG $(50 \mu \mathrm{M}): n=5$; LFS + DHPG $(50 \mu \mathrm{M}): n=5$. Sequential LFS and DHPG $(50 \mu \mathrm{M})$ did not increase ZX1 potentiation compared with LFS (not significant, $p=0.92)$ or DHPG $(50 \mu \mathrm{M})$ alone (not significant, $p=0.855)$. One-way ANOVA/Bonferroni.

baseline conditions. Together, these results suggest that postsynaptic rises in $\mathrm{Ca}^{2+}$ are necessary for Z-LTD.

To test whether rises in postsynaptic $\mathrm{Ca}^{2+}$ are sufficient to induce Z-LTD, we used CPA (20 $\mu \mathrm{M}, \mathrm{Ca}^{2+}$-ATPase inhibitor) to deplete $\mathrm{Ca}^{2+}$ stores from the endoplasmic reticulum and thus increase intracellular $\mathrm{Ca}^{2+}$. CPA application potentiated AMPAR EPSCs (Fig. 5D, black), and this potentiation was blocked by either prior ZX1 application (Fig. $5 D$, purple) or BAPTA in the pipette (Fig. 5D, orange). These results support that the CPA-induced potentiation of EPSCs is dependent on extracellular zinc and rises in postsynaptic $\mathrm{Ca}^{2+}$. This is consistent with the notion that the CPA-induced enhancement of AMPAR EPSCs is due to a CPA- induced Z-LTD-like mechanism leading to reduced zinc signaling and subsequent lack of inhibition of AMPARs by synaptically released zinc. Consistent with this hypothesis, incubation of slices with CPA eliminated ZX1 potentiation of EPSCs, presumably by inducing Z-LTD (Fig. 5F, blue), compared with interleaved control experiments without $\mathrm{CPA}$ incubation (Fig. $5 F$, red, and Fig. $5 G$, red vs blue). Finally, the effect of CPA on eliminating ZX1 potentiation (Fig. $5 F$, blue) was blocked when CPA was applied after BAPTA in the pipette, as evidenced by the restoration of subsequent ZX1 potentiation (Fig. $5 H$ ). This further supports that CPA-induced ZLTD, similarly to HFS-induced Z-LTD (Fig. $5 A$ ), requires rises in postsynaptic $\mathrm{Ca}^{2+}$. Together, although these experiments do not 
A
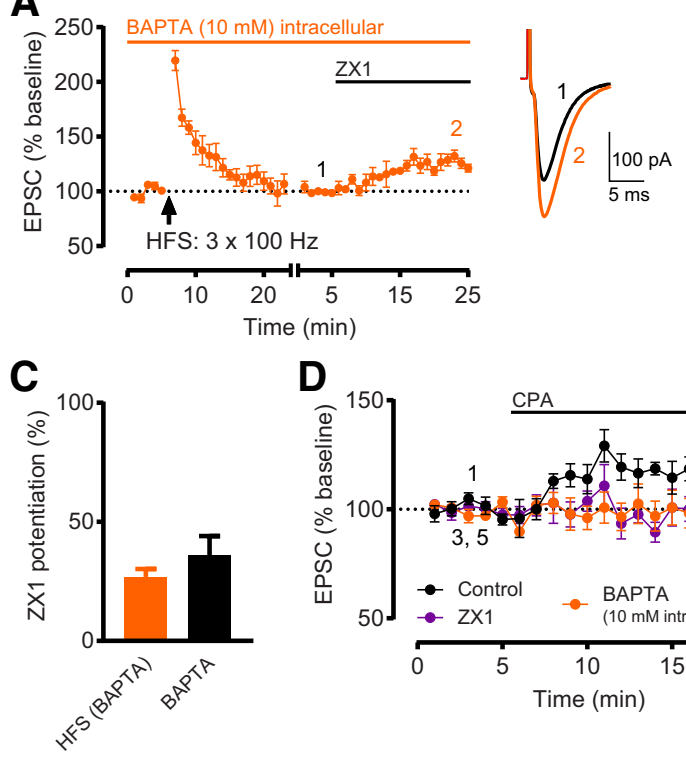

D

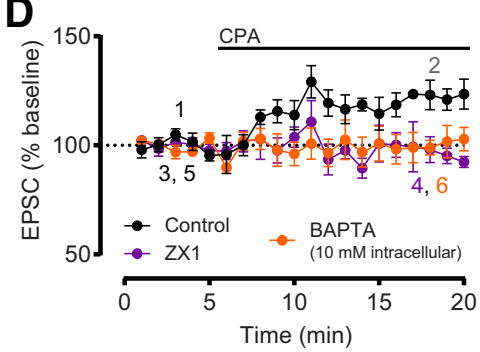

\begin{tabular}{cccccc}
\hline 0 & 5 & 10 & 15 & 20 & 25 \\
& &
\end{tabular}
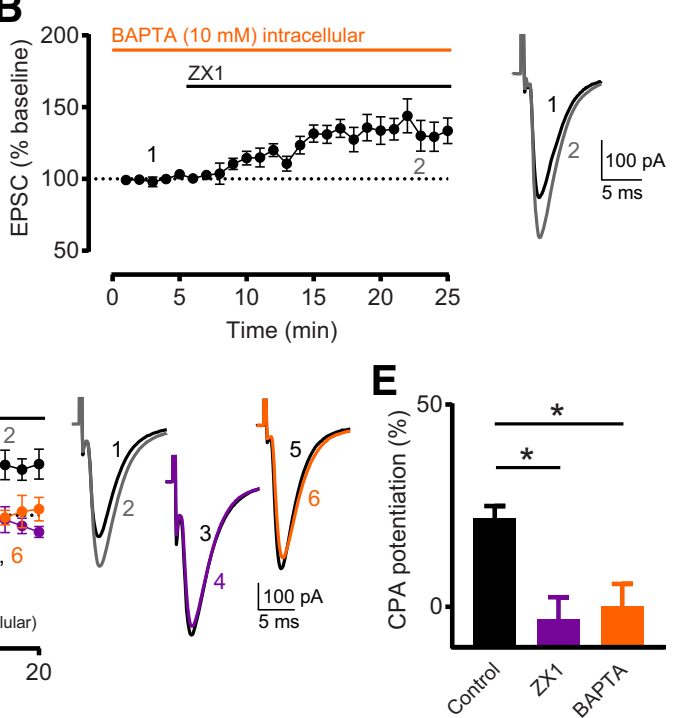

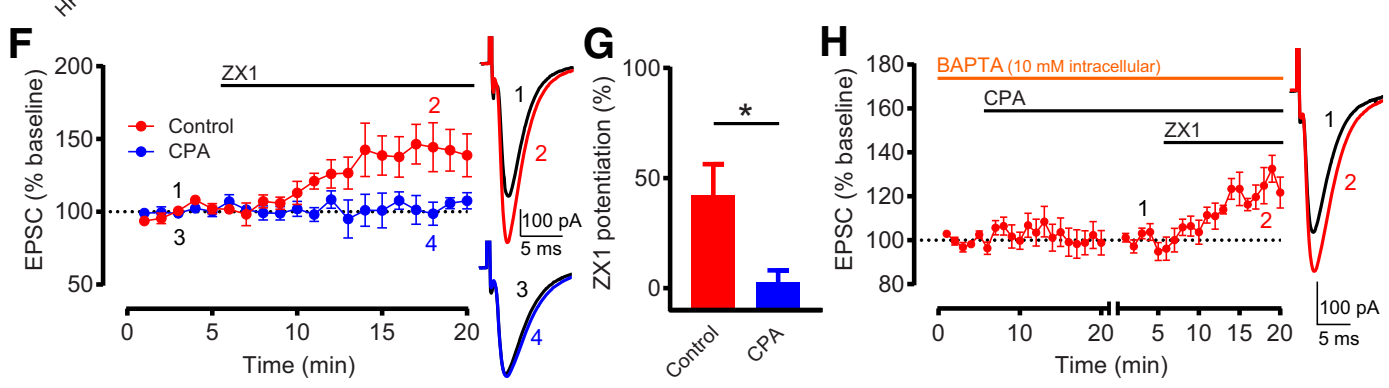

Figure 5. Rises in postsynaptic calcium are necessary and sufficient for inducing Z-LTD. $A$, Time course of AMPAR EPSC amplitude before and after HFS, and before and after subsequent ZX1 application, with intracellular recording solution containing $10 \mathrm{mM} \mathrm{BAPTA}$. HFS did not induce LTP $\left(n=7\right.$, not significant, $p=0.27$, paired $t$ test). ZX1 potentiated EPSCs ( $n=7$, ${ }^{*} p=0.0019$, paired $t$ test). To examine ZX1 potentiation after HFS, similar approach and renormalization as in Figure $1($ were performed. $B$, Time course of AMPAR EPSC amplitude before and after ZX1 application, normalized to baseline before ZX1 application, with intracellular recording solution containing $10 \mathrm{mM} \mathrm{BAPTA.} \mathrm{ZX1} \mathrm{potentiated} \mathrm{EPSCS} \mathrm{(} n=7,{ }^{*} p=0.0036$, paired $t$ test). $\boldsymbol{A}, \boldsymbol{B}$, Example traces represent AMPAR EPSCs before and after ZX1. C, Average ZX1 potentiation (\% increase from baseline) during the last 5 min of ZX1 application (minutes 21-25). HFS (BAPTA) $(n=7)$ versus BAPTA $(n=7)$ : not significant, $p=0.3106$, unpaired $t$ test. $\boldsymbol{D}$, Time course of AMPAR EPSC amplitude before and after CPA application $(20 \mu \mathrm{M})$, normalized to baseline before CPA application, in controls (black), in the presence of ZX1 (purple), and with BAPTA-containing intracellular recording solution (orange). In controls, CPA potentiated EPSCs ( $n=5$, ${ }^{*} p=0.004$, paired $t$ test). In the presence of ZX1, CPA did not potentiate EPSCS ( $n=4$, not significant, $p=0.40$, paired $t$ test). With intracellular BAPTA, CPA did not potentiate EPSCs ( $n=6$, not significant, $p=0.81$, paired $t$ test). Example traces represent AMPAR EPSCS before and after (PA application. $\boldsymbol{E}$, Average (PA potentiation (\% increase from baseline) during the last 5 min of CPA application (minutes 16-20). Compared with controls $(n=5)$, CPA potentiation was reduced in the presence of ZX1 $\left(n=4,{ }^{*} p=0.011\right)$, and with intracellular BAPTA ( $n=6$, $\left.{ }^{*} p=0.013\right)$. One-way ANOVA/Bonferroni. $\boldsymbol{F}$, Time course of AMPAR EPSC amplitude before and after ZX1 application, normalized to baseline before ZX1 application, in the presence (and prior incubation) of CPA (blue) and in controls (without CPA, red). In controls, ZX1 potentiated EPSCS ( $n=5,{ }^{*} p=0.036$, paired $t$ test). In CPA, ZX1 did not potentiate EPSCs ( $n=5$, not significant, $p=0.52$, paired $t$ test). Example traces represent AMPAR EPSCs before and after ZX1 application. $G$, Average ZX1 potentiation (\% increase from baseline) during the last 5 min of ZX1 application (minutes $16-$ 20). Control $(n=5)$ versus CPA $(n=5):{ }^{*} p=0.034$, unpaired $t$ test. $\boldsymbol{H}$, Time course of AMPAR EPSC amplitude before and after CPA application, and before and after subsequent $Z X 1$ application, with BAPTA-containing intracellular recording solution. After obtaining a stable baseline, CPA was applied. To examine ZX1 potentiation after CPA, after obtaining a stable baseline after CPA, AMPAR EPSC amplitude was renormalized to the new baseline before ZX1 application. The renormalization is indicated by a gap and restart of timing in the $x$ axis. With intracellular BAPTA, after CPA application, ZX1 potentiated EPSCS $\left(n=5,{ }^{*} p=0.0016\right.$, paired $t$ test). Example traces represent AMPAR EPSCs before and after ZX1 application.

support a definitive connection between postsynaptic $\mathrm{Ca}^{2+}$ and $\mathrm{G} 1$ mGluR signaling in Z-LTD, they clearly show that increases in postsynaptic $\mathrm{Ca}^{2+}$ are necessary and sufficient for Z-LTD (see Discussion).

Expression of G1 mGluR-dependent Z-LTD is mediated, at least in part, by reduced presynaptic zinc release

So far, we used activity-dependent changes in the amount of ZX1 potentiation of AMPAR EPSCs for assessing changes in synaptic zinc signaling (Z-LTP and Z-LTD). However, ZX1 potentiation is determined by the postsynaptic zinc-mediated inhibition of AMPAR EPSCs (Kalappa et al., 2015), as well as the amount of presynaptic zinc release. Because previous studies demonstrated sensory experience-dependent, long-term modulation of presynaptic zinc levels (Nakashima and Dyck, 2009; Kalappa et al., 2015), we tested whether synaptic zinc plasticity is expressed, at least in part, by activity-dependent changes in presynaptic zinc release. If zinc plasticity involves modulation of presynaptic zinc signaling, the induction of synaptic zinc plasticity should also affect postsynaptic NMDAR EPSCs, which are inhibited by direct high-affinity binding of zinc to the extracellular N-terminal domain of NMDARs and subsequent allosteric inhibition (Paoletti et al., 1997; Vergnano et al., 2014). To test this hypothesis, we quantified the ZX1 potentiation of NMDAR EPSCs after inducing Z-LTD with HFS. To monitor NMDAR EPSCs, we used a short train of presynaptic stimulation ( 5 pulses at $20 \mathrm{~Hz}$ ) to activate extrasynaptic NMDARs, for NMDAR EPSCs recorded in somata of CWCs are mostly mediated by extrasynaptic NMDARs activated by glutamate spillover during this short 

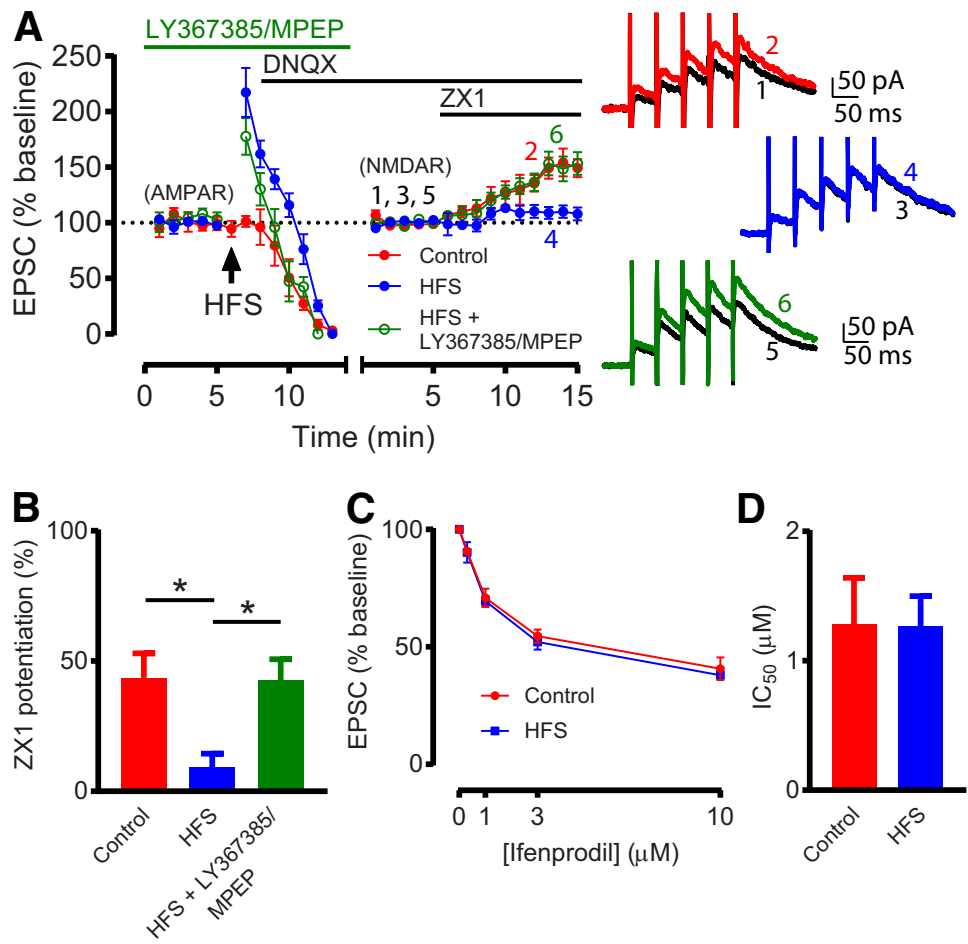

E
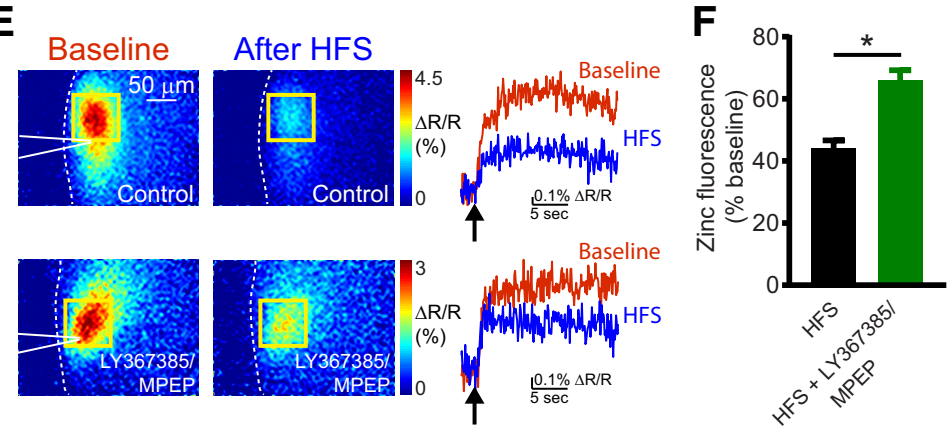

Figure 6. G1 mGluR-dependent Z-LTD reduces presynaptic zinc release and zinc inhibition of NMDARs. A, Left, Time course of AMPAR EPSC amplitude before and after HFS, and NMDAR EPSC amplitude before and after subsequent ZX1 application (blue); similar time course in control experiments (without HFS, red); and similar time course with HFS in the presence of LY367385 (100 $\mu \mathrm{M})$ and MPEP (4 $\mu \mathrm{M})$ (green). After obtaining a stable baseline of AMPAR EPSCS, HFS was delivered; then DNQX (20 $\mu \mathrm{M})$ was applied. NMDAR EPSCs were then recorded at $40 \mathrm{mV}$, normalized to the baseline NMDAR EPSC amplitude before ZX1 application. The switch from AMPAR to NMDAR EPSC time course, and the renormalization of EPSC amplitude are indicated by a gap and restart of timing in the $x$ axis. In controls, ZX1 potentiated NMDAR EPSCS $\left(n=5,{ }^{*} p=0.027\right.$, paired $t$ test). After HFS, ZX1 did not potentiate NMDAR EPSCS ( $n=6$, not significant, $p=0.16$, paired $t$ test). After HFS + LY367385/MPEP, ZX1 potentiated NMDAR EPSCS ( $n=5,{ }^{*} p=0.038$, paired $t$ test). Right, Example NMDAR EPSCs before and after $Z X 1$ application. $B$, Average $Z X 1$ potentiation (\% increase from baseline) during the last $5 \mathrm{~min}$ of $Z X 1$ application (minutes 11-15). Control: $n=5$; HFS: $n=6$; HFS + LY367385, MPEP: $n=5$. HFS reduced ZX1 potentiation compared with control ( $\left.{ }^{*} p=0.012\right)$; this reduction was blocked by LY367385 and MPEP ( $\left.{ }^{*} p=0.015\right)$. One-way ANOVA/Bonferroni. C, Doseresponse of NMDAR EPSCS (\% baseline) for increasing concentrations of ifenprodil, in controls (red) and after HFS (blue). Control: $n=3-5$ per concentration; HFS: $n=3$ or 4 per concentration. $\boldsymbol{D}, I_{50}$ of ifenprodil, from dose-responses in $\boldsymbol{C}$. Not significant, $p=0.97$, comparison of fits, extra sum-of-squares $F$ test. $\boldsymbol{E}$, Top left, Representative stimulus-evoked (50 pulses, $100 \mathrm{~Hz})$ zinc-mediated fluorescent signals using LZ9 $(2 \mu \mathrm{M})$, before and after HFS. Dotted white line outlines the edge of the DCN slice. White triangle represents the stimulating electrode. Yellow square represents the ROI. Top right, Time course of representative ratiometric fluorescent signals, before and after HFS. Arrow indicates the time of stimulation. HFS significantly reduced stimulus-evoked zinc fluorescence (maximum $\Delta \mathrm{R} / \mathrm{R})\left(n=6,{ }^{*} p=0.0002\right.$, paired $t$ test). Bottom, Same as above, but in the presence of LY367385 $(100 \mu \mathrm{M})$ and MPEP $(4 \mu \mathrm{M}) . \boldsymbol{F}$, Average stimulus-evoked zinc fluorescence (maximum $\Delta \mathrm{R}$ / R) after HFS, normalized to baseline before HFS. Compared with HFS alone $(n=6)$, LY367385/MPEP $(n=6)$ significantly attenuated the HFS-induced reduction of zinc fluorescence $\left({ }^{*} p=0.0005\right.$, unpaired $t$ test).

train (Anderson et al., 2015). Moreover, at $20 \mathrm{~Hz}$ stimulation, ZX1 does not potentiate NMDAR EPSCs in ZnT3 KO mice, confirming that these NMDAR EPSCs are inhibited by ZnT3-dependent, synaptically released zinc, but not tonic zinc (Anderson et al., 2015). To avoid keeping CWCs at $40 \mathrm{mV}$ for too long while recording NMDAR EPSCs, and to maintain the same induction protocol used in our previous experiments, we initially recorded AMPAR EPSCs at $-70 \mathrm{mV}$ and then applied HFS (Fig. 6A). Subsequently, we blocked AMPARs with DNQX (20 $\mu \mathrm{M}, \mathrm{AMPA} /$ kainate receptor antagonist) and recorded at $40 \mathrm{mV}$ to obtain a stable baseline of NMDAR EPSCs before applying ZX1 (Fig. 6A). Consistent with our results on AMPAR EPSCs, HFS significantly reduced $\mathrm{ZX} 1$ potentiation of NMDAR EPSCs (Fig. 6A, blue) compared with control experiments where HFS was not applied (Fig. 6A, red, and Fig. 6B, blue vs red). These results suggest that $\mathrm{Z}$ LTD reduces zinc-mediated inhibition of NMDARs. To determine whether this plasticity shares the same mechanism as Z-LTD evidenced by changes in the ZX1 potentiation of AMPAR EPSCs, we tested whether G1 mGluR activation is required. Indeed, in the presence of LY367385/MPEP, we observed ZX1 potentiation of NMDAR EPSCs after HFS (Fig. 6A, green, and Fig. 6B, green vs blue), demonstrating that G1 mGluR activation is necessary for Z-LTD assessed by NMDAR EPSCs. Together, our results suggest that G1 mGluRdependent synaptic zinc plasticity modulates zinc-mediated inhibition of AMPARs and NMDARs similarly. Moreover, this finding suggests that Z-LTD is independent of the postsynaptic target, consistent with the notion that Z-LTD is expressed by reduced presynaptic zinc release.

We next tested whether activity-dependent changes in postsynaptic NMDAR subunit composition modulate zinc sensitivity during Z-LTD. NMDARs are composed of two GluN1 subunits and two GluN2 subunits (Traynelis et al., 2010). GluN2A-containing NMDARs (GluN1/GluN2A diheteromers and GluN2/ GluN2A/GluN2B triheteromers) have nanomolar affinity for zinc, whereas GluN1/ GluN2B diheteromers have micromolar affinity (Paoletti et al., 1997; Rachline et al., 2005; Tovar and Westbrook, 2012; Hansen et al., 2014). Therefore, the reduced zincmediated inhibition of NMDAR EPSCs after HFS (Fig. $6 A, B$ ) could be explained either by a reduction of presynaptic zinc release or an increase in the proportion of GluN2B NMDAR subunits. To test for a potential change in NMDAR subunit composition, we investigated whether HFS increases the sensitivity of NMDAR EPSCs to ifenprodil, a GluN2B-selective antagonist (Fig. 6C,D) (Tovar and Westbrook, 2012; Hansen et al., 2014). Compared with control experiments (without HFS), HFS did not affect the ifenprodil sensitivity $\left(\mathrm{IC}_{50}\right)$ of NMDAR EPSCs (Fig. $6 C, D$ ). This indicates that HFS-induced plasticity does not alter the proportions of GluN2B 
A
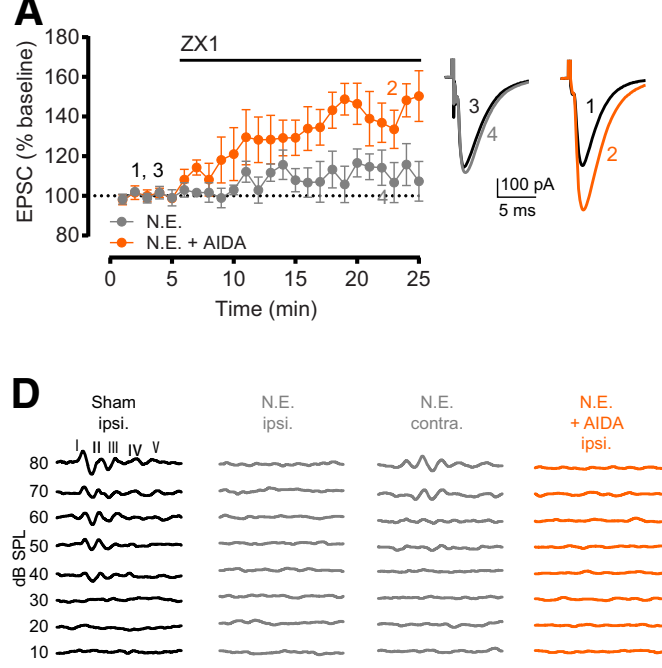
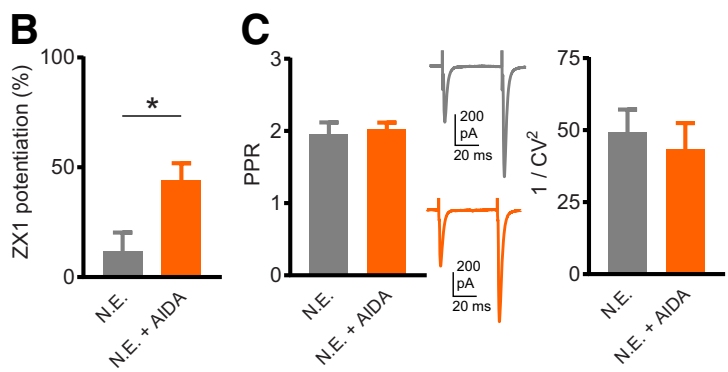

$\mathbf{E}$
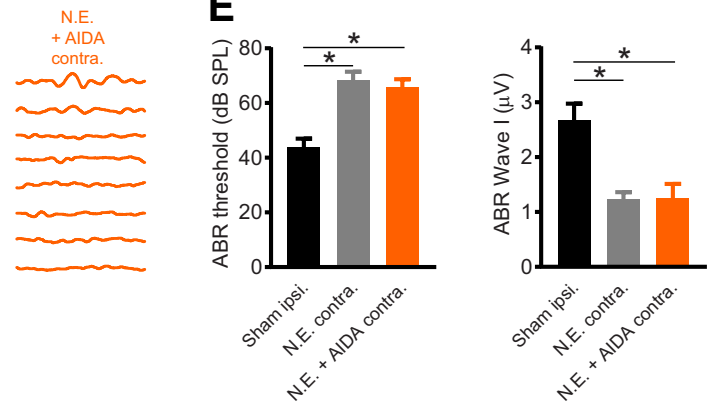

Figure 7. Sound-induced Z-LTD requires G1 mGluR activation. $\boldsymbol{A}$, Time course of AMPAR EPSC amplitude before and after ZX1 application in slices from NE mice (gray) and NE AIDA-treated mice (orange). In NE mice, ZX1 did not potentiate EPSCS $\left(n=5\right.$, not significant, $p=0.23$, paired $t$ test). In NE AIDA-treated mice, ZX1 potentiated EPSCS $\left(n=6,{ }^{*} p=0.005\right.$, paired $t$ test). Example traces represent AMPAR EPSCs before and after ZX1. B, Average ZX1 potentiation (\% increase from baseline) during the last 5 min of ZX1 application (minutes 21-25). NE ( $n=5$ ) versus $\mathrm{NE}+\operatorname{AIDA}(n=6):{ }^{*} p=0.024$, unpaired $t$ test. $C$, Left, Average PPR (pulse 2/pulse 1) of baseline AMPAR EPSCs in slices from NE mice and NE AIDA-treated mice. NE ( $\left.n=6\right)$ versus NE + AIDA ( $n=8)$ : not significant, $p=0.70$, unpaired $t$ test. Example traces represent AMPAR EPSCs in response to two pulses. Right, CV analysis $\left(1 / \mathrm{CV}^{2}\right)$ of baseline AMPAR EPSCs (pulse 1$)$ in slices from NE mice and NE AIDA-treated mice. NE $(n=6)$ versus NE + AIDA $(n=8)$ : not significant, $p=0.49$, Mann-Whitney test. $D$, Example ABRs $(10-80 \mathrm{~dB}$ SPL sound stimuli) from shamexposed mice (recorded from sham-exposed, ipsilateral ear, black), NE mice (gray), and NE AIDA-treated mice (orange). Because no ABRs were detected in the ipsilateral ears of NE mice, ABRs were measured from ears contralateral to noise exposure. $\boldsymbol{E}$, Left, Average ABR thresholds (dB SPL). Sham ipsilateral: $n=8$; NE contralateral: $n=6$; NE + AIDA contralateral: $n=7$. NE increased ABR thresholds compared with sham-exposed $\left({ }^{*} p=0.0002\right)$, but AIDA and NE did not affect increases in ABR thresholds compared with NE alone, Kruskal-Wallis test/Dunn. Right, Average ABR Wave I amplitude $(\mu \mathrm{V})$. Sham ipsilateral: $n=8$; NE contralateral: $n=6$; NE + AIDA contralateral: $n=7$. NE decreased ABR Wave I amplitude compared with sham-exposed $\left({ }^{*} p=0.0024\right)$, but AIDA and NE did not affect decreases in ABR Wave I amplitude compared with NE alone, Kruskal-Wallis test/Dunn.

versus GluN2A NMDAR subunits, further suggesting that Z-LTD is not due to reduced zinc sensitivity caused by a decrease in the relative contribution of GluN2A versus GluN2B in the NMDAR EPSC. Therefore, these results support that Z-LTD is likely expressed by reduced presynaptic zinc release, rather than postsynaptic receptor or signaling modifications.

To test directly whether reduced presynaptic zinc release is associated with Z-LTD expression, we quantified evoked zinc release in DCN slices. To do this, we used the extracellular ratiometric zinc sensor LZ9 $(2 \mu \mathrm{M})$, which is composed of a zincinsensitive red fluorophore linked to a zinc-sensitive green fluorophore (Anderson et al., 2015; Kalappa et al., 2015). LZ9 is ideal for measuring synaptic zinc release because the ratiometric approach (see Materials and Methods) is independent of sensor concentration and controls for instrument variability and background noise (Anderson et al., 2015). We measured zinc-mediated fluorescence in response to PF electrical stimulation before and after HFS (Fig. 6E). We found that evoked zinc release was significantly reduced after HFS (Fig. 6E,F). This result is consistent with the notion that Z-LTD is associated with a reduction in synaptic zinc release. To determine whether this reduction shares the same mechanism as Z-LTD, we tested whether G1 mGluR activation is required. Indeed, the presence of LY367385/MPEP significantly attenuated the HFS-induced reduction of zinc release (Fig. 6E,F). Although G1 mGluR antagonists did not eliminate the HFS-induced reduction of zinc fluorescence (see Discussion), this result demonstrates that G1 mGluR activation contributes to the HFS-induced reduction of zinc release, which is consistent with our electrophysiological results. Together, these results strongly support that the expression mechanisms of
Z-LTD are mediated, at least in part, by activity-dependent reductions in presynaptic zinc release.

\section{Sound-induced G1 mGluR-dependent Z-LTD}

Our experiments described here, using in vitro brain slice electrophysiology in the DCN, point toward a mechanism of bidirectional long-term synaptic zinc plasticity dependent on G1 mGluR activation. We therefore hypothesized that G1 mGluR activation may also be necessary for the reduction in synaptic zinc signaling observed in the DCN after exposure to loud sound (Kalappa et al., 2015). To test this hypothesis, we quantified the ZX1 potentiation of PF EPSCs in DCN slices from mice exposed to loud sound $(116 \mathrm{~dB}, 4 \mathrm{~h})$. Our previous studies demonstrated that noise exposure, identical to the one used here, reduces presynaptic zinc levels, synaptic zinc release, and zinc-mediated inhibition of AMPARs (Kalappa et al., 2015). Consistent with these findings, we did not observe ZX1 potentiation in slices from NE mice (Fig. 7A, gray), demonstrating the sound-induced reduction of zinc signaling (Z-LTD). To test whether G1 mGluRs are necessary for this Z-LTD in slices from NE mice, we administered a systemic, blood-brain barrier-permeable G1 mGluR antagonist (AIDA, i.p., $2 \mathrm{mg} / \mathrm{kg}$; twice: $30 \mathrm{~min}$ before and $1.5 \mathrm{~h}$ after beginning the noise exposure). Indeed, we observed $\mathrm{ZX} 1$ potentiation in slices from NE mice treated with AIDA (Fig. 7A, orange, and Fig. $7 B$, orange vs gray), suggesting that in vivo inhibition of G1 mGluR activity blocked the sound-induced Z-LTD.

Although AIDA treatment blocked Z-LTD in DCN PF synapses (Fig. $7 A, B$ ), it did not affect assays that are sensitive to presynaptic glutamate release probability, such as PPR and CV 
analysis (Fig. 7C). This indicates that sound-induced G1 mGluRdependent Z-LTD specifically modulates synaptic zinc signaling, without affecting presynaptic glutamate release in PFs. Furthermore, AIDA treatment did not affect sound-induced hearing loss in NE mice, quantified with ABRs (Fig. 7D). ABRs reflect the synchronous activity, arising from the auditory nerve (Wave I), of auditory brainstem nuclei to the inferior colliculus (Waves II$\mathrm{V})$ in response to sound stimuli. Elevated ABR thresholds indicate increased hearing thresholds. However, similar ABR thresholds may be accompanied by differences in the suprathreshold response of Wave I, which could reflect differential degeneration of the auditory nerve (Kujawa and Liberman, 2009). AIDA treatment did not affect noise-induced changes in either ABR thresholds or Wave I amplitude (Fig. 7E), thus indicating that the effect of AIDA on blocking Z-LTD is not due to differential noiseinduced hearing loss after AIDA treatment. Together, these results demonstrate that sound-induced Z-LTD requires G1 mGluR activation, consistent with our in vitro results.

\section{Discussion}

Our results show that long-term synaptic zinc plasticity is an experience- and G1 mGluR-dependent mechanism that bidirectionally modulates synaptic zinc signaling in the DCN. Whereas the exact synaptic, natural, and ethologically relevant stimuli that elicit Z-LTP and Z-LTD remain unknown, here we developed in vitro and in vivo models to study Z-LTP and Z-LTD. This is a crucial step toward further elucidation of the detailed natural stimuli eliciting long-term synaptic zinc plasticity, as well as the precise cellular and molecular mechanisms underlying the induction and expression of Z-LTP and Z-LTD.

\section{Mechanisms of G1 mGluR-dependent Z-LTP and Z-LTD}

Our results show that differential activation of G1 mGluRs, by either LFS/HFS or high/low concentrations of DHPG, determines the induction and direction of long-term synaptic zinc plasticity. Prolonged LFS causes Z-LTP, similarly to G1 mGluR activation with $50 \mu \mathrm{M}$ DHPG; whereas brief HFS causes Z-LTD, similarly to activation with $5 \mu \mathrm{M}$ DHPG. Although the precise dynamics of mGluR activation during LFS and HFS are unknown, these results are consistent with the notion that prolonged LFS may lead to greater G1 mGluR activation than brief HFS.

Our results demonstrate that rises in postsynaptic $\mathrm{Ca}^{2+}$ are necessary and sufficient for the induction of Z-LTD (Fig. 5). While our results do not establish a definitive connection between these postsynaptic $\mathrm{Ca}^{2+}$ changes and G1 mGluR activation, the role of $\mathrm{Ca}^{2+}$ identified here is similar to the role of intracellular $\mathrm{Ca}^{2+}$ shown in previous studies on G1 mGluR-dependent synaptic plasticity. Namely, in the hippocampus, LFS induces G1 mGluR-mediated LTD via postsynaptic AMPAR endocytosis involving $\mathrm{Ca}^{2+}$ release from endoplasmic reticulum stores and dendritic protein synthesis (Huber et al., 2000; Holbro et al., 2009; Luscher and Huber, 2010; Pick and Ziff, 2018). Moreover, HFS or theta-burst stimulation induces G1 mGluR-mediated LTP, also involving endoplasmic reticulum $\mathrm{Ca}^{2+}$ release, resulting in postsynaptic AMPAR/ NMDAR trafficking or enhanced presynaptic glutamate release (Topolnik et al., 2006; Wu et al., 2008; Anwyl, 2009). We found that G1 mGluR activation is necessary and sufficient for both Z-LTD and Z-LTP (Figs. 2-4). We showed a critical role of postsynaptic $\mathrm{Ca}^{2+}$ in Z-LTD (Fig. 5), but it remains unknown whether a similar $\mathrm{Ca}^{2+}$-dependent mechanism also underlies Z-LTP. It also remains unknown whether G1 mGluR-dependent Z-LTP and Z-LTD are downstream effects of the same signaling pathways that induce
LTD and LTP, or occur through separate mechanisms. Future experiments will be needed to dissect the detailed mechanisms of G1 mGluR activation and postsynaptic $\mathrm{Ca}^{2+}$ dynamics in zinc plasticity, and whether or how they may interact with known G1 mGluR-dependent signaling pathways.

\section{Interpretation of LZ9 imaging and electrophysiological experiments}

We found that HFS-induced Z-LTD cannot be explained by postsynaptic changes in the relative contributions of GluN2A versus GluN2B subunits in NMDAR EPSCs (Fig. 6C,D). Although our experiments do not exclude potential contributions of postsynaptic mechanisms in the expression of Z-LTD, our results support that Z-LTD is mediated, at least in part, by activity-dependent modulation of presynaptic zinc release (Fig. $6 A, B, E, F)$.

In our imaging experiments, we found that G1 mGluRs contribute to the HFS-induced reduction of zinc release (Fig. 6E,F); however, G1 mGluR antagonists did not entirely block the HFSinduced reduction of zinc release. This suggests that additional G1 mGluR-independent mechanisms might contribute to HFSinduced reductions of zinc release in DCN slices. For example, it has been previously suggested that high levels of synaptic activity may result in a passive depletion of vesicular zinc content (McAllister and Dyck, 2017). Another possibility is that the G1 mGluR-independent reductions of zinc release may be due to the rundown of the fluorescent signal after each successive stimulation (data not shown). Namely, because LZ9 binds synaptic zinc with high affinity, it will not necessarily release the bound zinc unless a higher-affinity chelator is added to unbind the zinc from LZ9. Therefore, it is likely that, after each stimulation, there is slightly less available unbound LZ9 to respond to the next stimulation, leading to a slight reduction in the normalized fluorescence response $(\Delta R / R)$. For this reason, in these experiments, we did not give many rounds of stimulation (see Materials and Methods). Moreover, the fact that LZ9 binds zinc to produce fluorescence, and this zinc remains bound, also explains why the stimulus-evoked fluorescent signals do not return to baseline (Fig. 6E) (Anderson et al., 2015; Kalappa et al., 2015). Although it is possible that rundown, or other G1 mGluR-independent mechanisms, could contribute in part to the decreased fluorescent signals after HFS, importantly the amount of this decrease is significantly attenuated by LY367385/MPEP (Fig. 6E,F). This result suggests that G1 mGluR activation contributes to the HFSinduced reduction of zinc release. This conclusion is consistent with our electrophysiological results demonstrating that G1 mGluR antagonists eliminated Z-LTD (Figs. 2, 6). Together, our experiments identify a critical role of G1 mGluR activation in synaptic zinc plasticity; this plasticity involves activity-dependent changes in presynaptic zinc release.

\section{Implications of Z-LTP and Z-LTD for short-term plasticity}

Previous studies in DCN PF synapses revealed that synaptic zinc triggers endocannabinoid synthesis, which inhibits presynaptic glutamate release and modulates short-term plasticity (PerezRosello et al., 2013; Kalappa and Tzounopoulos, 2017). During high-frequency $(50 \mathrm{~Hz})$ trains, synaptic zinc inhibits AMPAR EPSCs during the first few stimuli but enhances steady-state EPSCs in subsequent stimuli by recruiting endocannabinoid signaling and enhancing synaptic facilitation (Kalappa and Tzounopoulos, 2017). Therefore, long-term increases in zinc signaling (Z-LTP) would enhance endocannabinoid activation during subsequent stimulus trains, increase synaptic facilitation, and 
further enhance steady-state EPSCs. Conversely, long-term decreases in zinc signaling, via Z-LTD, would reduce endocannabinoid activation, decrease synaptic facilitation, and suppress steady-state EPSCs.

Following stimulus trains, zinc-mediated endocannabinoid activation causes short-term depression and inhibits short-term facilitation (Perez-Rosello et al., 2013). Therefore, Z-LTP and ZLTD are expected to shift the balance between short-term facilitation and short-term depression in DCN synapses. Z-LTP will enhance subsequent zinc-mediated short-term depression, whereas Z-LTD will enhance short-term facilitation. Together, our results highlight a powerful mechanism by which long-term bidirectional zinc plasticity may modulate short-term glutamatergic synaptic plasticity.

\section{Implications of Z-LTP and Z-LTD for LTD and LTP}

In central synapses, including DCN PF synapses, the direction and size of LTP or LTD are determined by the combination of multiple simultaneous LTP and LTD mechanisms (O'Connor et al., 2005; Bender et al., 2006; Tzounopoulos et al., 2007; Shen et al., 2008; Zhao and Tzounopoulos, 2011). Our results suggest that Z-LTD, by reducing zinc-mediated inhibition of AMPAR EPSCs and thus enhancing baseline synaptic strength, is a new mechanism of LTP. Conversely, Z-LTP, by enhancing zincmediated inhibition of AMPAR EPSCs, is a new mechanism of LTD. In DCN PF synapses, LTP and LTD are influenced by the coactivation of presynaptic and postsynaptic signaling mechanisms, including NMDARs, mGluRs, muscarinic acetylcholine receptors, and endocannabinoid signaling (Fujino and Oertel, 2003; Tzounopoulos et al., 2007; Zhao and Tzounopoulos, 2011). Therefore, bidirectional zinc plasticity likely acts together with these other known mechanisms to shape the size and direction of synaptic plasticity.

Several of our results are consistent with this notion. Z-LTD contributes to HFS-induced NMDAR-independent LTP (Fig. $2 A, B)$; however, a G1 mGluR-independent glutamate-based mechanism mediates LTP when Z-LTD is blocked (Fig. 2C). Moreover, G1 mGluR activation with $5 \mu \mathrm{M}$ DHPG induced ZLTD, but did not induce LTP, due to a concurrent synaptic depression that counterbalances the LTP effect of Z-LTD (Fig. $4 A, E)$. LFS induced Z-LTP, but did not induce LTD, due to a compensatory LTP that counterbalances the LTD effect of ZLTP (Fig. $3 A, B$ ). Together, all our results are consistent with previous studies showing that LTP and LTD mechanisms occur simultaneously in DCN PF synapses (Tzounopoulos et al., 2007). Future studies will be needed to determine the precise interaction of zinc- and glutamate-based plasticity mechanisms. A relevant consideration is whether zinc is sequestered in the glutamatecontaining vesicles or in a separate pool. Previous studies showed that ZnT3 (vesicular zinc transporter) and VGlut1 (vesicular glutamate transporter) interact, leading to the coloading of both zinc and glutamate in zinc-rich vesicles (Salazar et al., 2004, 2005). However, not all glutamate-containing vesicles also contain zinc: the zinc-rich vesicle pool comprises a subset of vesicles in presynaptic glutamatergic terminals (Salazar et al., 2004), and is preferentially released during higher levels of neuronal activity (Lavoie et al., 2011).

Together, our results provide a novel framework for integrating zinc-based plasticity (Z-LTP and Z-LTD) with different forms of glutamate-based plasticity (LTP and LTD). Importantly, our results add Z-LTP and Z-LTD as new mechanisms of LTD and LTP at zinc-containing glutamatergic synapses.
In addition to DCN PF synapses, we propose, although not tested here, that Z-LTP and Z-LTD may contribute to LTD and LTP in other synaptic zinc-containing brain areas, which express G1 mGluR-dependent LTD and LTP, such as the hippocampus, amygdala, and striatum (Oliet et al., 1997; Huber et al., 2000; Gubellini et al., 2003; Topolnik et al., 2006; Wu et al., 2008; Anwyl, 2009; Luscher and Huber, 2010; Chen et al., 2017; McAllister and Dyck, 2017). In the hippocampus, LFS induces G1 mGluR-mediated LTD, whereas HFS induces LTP (Oliet et al., 1997; Huber et al., 2000; Topolnik et al., 2006; Wu et al., 2008; Anwyl, 2009). Therefore, LFS-induced Z-LTP would likely further enhance the effects of G1 mGluR-LTD, by increasing zinc inhibition of AMPARs, whereas HFS-induced Z-LTD would further enhance the effects of LTP, by reducing zinc inhibition of AMPARs. Thus, synaptic zinc plasticity likely serves as a positive feedback mechanism to enhance the effects of G1 mGluR-dependent LTP or LTD on glutamatergic synaptic transmission.

\section{Implications of Z-LTP and Z-LTD for metaplasticity}

The induction of Z-LTP and Z-LTD is NMDAR-independent (Figs. 2, 3). However, zinc inhibits NMDARs and thus modulates the induction of NMDAR-dependent LTP and LTD in the hippocampus (Izumi et al., 2006; Takeda et al., 2009; Vergnano et al., 2014). As such, long-term synaptic zinc plasticity may contribute to "metaplasticity," the modulation of subsequent LTP and LTD (Abraham and Tate, 1997). Z-LTD, by reducing the inhibitory effect of zinc on NMDARs, may promote subsequent NMDAR-dependent LTP and decrease subsequent NMDARdependent LTD. Conversely, Z-LTP, by enhancing the inhibitory effect of zinc on NMDARs, may promote subsequent NMDAR-LTD over NMDAR-LTP. Therefore, zinc plasticity may serve as a positive feedback mechanism for NMDAR-dependent metaplasticity.

Synaptic zinc contributes to mossy fiber presynaptic LTP in response to HFS, via activation of TrkB receptors (Huang et al., 2008; Pan et al., 2011). Therefore, if HFS induces Z-LTD in mossy fiber synapses, it would act as a negative feedback mechanism by reducing subsequent LTP induction. Together, we propose that the role of Z-LTD and Z-LTP in LTP and LTD depends on the specific mechanisms underlying LTP and LTD, but overall, Z-LTD and Z-LTP likely act as positive feedback mechanisms to enhance G1 mGluR-dependent LTP and LTD, and NMDAR-dependent metaplasticity.

\section{Clinical and translational implications of zinc plasticity}

In the context of zinc plasticity as a positive feedback signal for NMDAR-dependent metaplasticity, it is interesting that exposure to loud sound, known to induce tinnitus, causes Z-LTD in the DCN. Although not tested here, it is possible that Z-LTD could potentially lead to runaway excitation due to enhanced LTP and decreased LTD, and thus to pathologic DCN hyperactivity associated with tinnitus (Tzounopoulos, 2008). Noiseinduced pathologic hyperexcitability through LTP/LTD-like mechanisms in the DCN PF synapses has been hypothesized and recently implicated in tinnitus treatment (Tzounopoulos, 2008; Marks et al., 2018), therefore suggesting that noise-induced reductions in synaptic zinc might contribute to tinnitus.

\section{References}

Abdul-Ghani MA, Valiante TA, Carlen PL, Pennefather PS (1996) Metabotropic glutamate receptors coupled to IP3 production mediate inhibition of IAHP in rat dentate granule neurons. J Neurophysiol 76:2691-2700. 
Abraham WC, Tate WP (1997) Metaplasticity: a new vista across the field of synaptic plasticity. Prog Neurobiol 52:303-323.

Anderson CT, Kumar M, Xiong S, Tzounopoulos T (2017) Cell-specific gain modulation by synaptically released zinc in cortical circuits of audition. Elife 6:e29893.

Anderson CT, Radford RJ, Zastrow ML, Zhang DY, Apfel UP, Lippard SJ, Tzounopoulos T (2015) Modulation of extrasynaptic NMDA receptors by synaptic and tonic zinc. Proc Natl Acad Sci USA 112:E2705-E2714.

Anwyl R (2009) Metabotropic glutamate receptor-dependent long-term potentiation. Neuropharmacology 56:735-740.

Auerbach BD, Rodrigues PV, Salvi RJ (2014) Central gain control in tinnitus and hyperacusis. Front Neurol 5:206.

Bender VA, Bender KJ, Brasier DJ, Feldman DE (2006) Two coincidence detectors for spike timing-dependent plasticity in somatosensory cortex. J Neurosci 26:4166-4177.

Bilak SR, Morest DK (1998) Differential expression of the metabotropic glutamate receptor mGluRlalpha by neurons and axons in the cochlear nucleus: in situ hybridization and immunohistochemistry. Synapse 28:251270.

Brown CE, Dyck RH (2002) Rapid, experience-dependent changes in levels of synaptic zinc in primary somatosensory cortex of the adult mouse. J Neurosci 22:2617-2625.

Brown CE, Dyck RH (2005) Modulation of synaptic zinc in barrel cortex by whisker stimulation. Neuroscience 134:355-359.

Chen A, Hu WW, Jiang XL, Potegal M, Li H (2017) Molecular mechanisms of group I metabotropic glutamate receptor mediated LTP and LTD in basolateral amygdala in vitro. Psychopharmacology (Berl) 234:681-694.

Cole TB, Wenzel HJ, Kafer KE, Schwartzkroin PA, Palmiter RD (1999) Elimination of zinc from synaptic vesicles in the intact mouse brain by disruption of the ZnT3 gene. Proc Natl Acad Sci USA 96:1716-1721.

Conn PJ, Pin JP (1997) Pharmacology and functions of metabotropic glutamate receptors. Annu Rev Pharmacol Toxicol 37:205-237.

Danscher G, Stoltenberg M (2005) Zinc-specific autometallographic in vivo selenium methods: tracing of zinc-enriched (ZEN) terminals, ZEN pathways, and pools of zinc ions in a multitude of other ZEN cells. J Histochem Cytochem 53:141-153.

Dyck RH, Chaudhuri A, Cynader MS (2003) Experience-dependent regulation of the zincergic innervation of visual cortex in adult monkeys. Cereb Cortex 13:1094-1109.

Frederickson CJ, Howell GA, Haigh MD, Danscher G (1988) Zinc-containing fiber systems in the cochlear nuclei of the rat and mouse. Hear Res 36:203-211.

Frederickson CJ, Koh JY, Bush AI (2005) The neurobiology of zinc in health and disease. Nat Rev Neurosci 6:449-462.

Fujino K, Oertel D (2003) Bidirectional synaptic plasticity in the cerebellumlike mammalian dorsal cochlear nucleus. Proc Natl Acad Sci USA 100:265-270.

Gubellini P, Saulle E, Centonze D, Costa C, Tropepi D, Bernardi G, Conquet F, Calabresi P (2003) Corticostriatal LTP requires combined mGluR1 and mGluR5 activation. Neuropharmacology 44:8-16.

Hansen KB, Ogden KK, Yuan H, Traynelis SF (2014) Distinct functional and pharmacological properties of triheteromeric GluN1/GluN2A/GluN2B NMDA receptors. Neuron 81:1084-1096.

Holbro N, Grunditz A, Oertner TG (2009) Differential distribution of endoplasmic reticulum controls metabotropic signaling and plasticity at hippocampal synapses. Proc Natl Acad Sci USA 106:15055-15060.

Huang YZ, Pan E, Xiong ZQ, McNamara JO (2008) Zinc-mediated transactivation of TrkB potentiates the hippocampal mossy fiber-CA3 pyramid synapse. Neuron 57:546-558.

Huber KM, Kayser MS, Bear MF (2000) Role for rapid dendritic protein synthesis in hippocampal mGluR-dependent long-term depression. Science 288:1254-1257.

Huber KM, Roder JC, Bear MF (2001) Chemical induction of mGluR5- and protein synthesis-dependent long-term depression in hippocampal area CA1. J Neurophysiol 86:321-325.

Izumi Y, Auberson YP, Zorumski CF (2006) Zinc modulates bidirectional hippocampal plasticity by effects on NMDA receptors. J Neurosci 26:7181-7188.

Kalappa BI, Tzounopoulos T (2017) Context-dependent modulation of excitatory synaptic strength by synaptically released zinc. eNeuro 4 : ENEURO.0011-17.2017
Kalappa BI, Anderson CT, Goldberg JM, Lippard SJ, Tzounopoulos T (2015) AMPA receptor inhibition by synaptically released zinc. Proc Natl Acad Sci USA 112:15749-15754.

Kim CH, Lee J, Lee JY, Roche KW (2008) Metabotropic glutamate receptors: phosphorylation and receptor signaling. J Neurosci Res 86:1-10.

Kouvaros S, Kumar M, Tzounopoulos T (2020) Synaptic zinc enhances inhibition mediated by somatostatin, but not parvalbumin, cells in mouse auditory cortex. Cereb Cortex Advance online publication. Retrieved February 24, 2020. doi: 10.1093/cercor/bhaa005.

Kujawa SG, Liberman MC (2009) Adding insult to injury: cochlear nerve degeneration after "temporary" noise-induced hearing loss. J Neurosci 29:14077-14085.

Kumar M, Xiong S, Tzounopoulos T, Anderson CT (2019) Fine control of sound frequency tuning and frequency discrimination acuity by synaptic zinc signaling in mouse auditory cortex. J Neurosci 39:854-865.

Lavoie N, Jeyaraju DV, Peralta MR, Seress L, Pellegrini L, Tóth K (2011) Vesicular zinc regulates the $\mathrm{Ca}^{2+}$ sensitivity of a subpopulation of presynaptic vesicles at hippocampal mossy fiber terminals. J Neurosci 31:18251-18265.

Li Y, Andereggen L, Yuki K, Omura K, Yin Y, Gilbert HY, Erdogan B, Asdourian MS, Shrock C, de Lima S, Apfel UP, Zhuo Y, Hershfinkel M, Lippard SJ, Rosenberg PA, Benowitz L (2017) Mobile zinc increases rapidly in the retina after optic nerve injury and regulates ganglion cell survival and optic nerve regeneration. Proc Natl Acad Sci USA 114:E209E218.

Luscher C, Huber KM (2010) Group 1 mGluR-dependent synaptic longterm depression: mechanisms and implications for circuitry and disease. Neuron 65:445-459.

Malenka RC, Nicoll RA (1993) NMDA-receptor-dependent synaptic plasticity: multiple forms and mechanisms. Trends Neurosci 16:521-527.

Malenka RC, Bear MF (2004) LTP and LTD: an embarrassment of riches. Neuron 44:5-21.

Manis PB, Spirou GA, Wright DD, Paydar S, Ryugo DK (1994) Physiology and morphology of complex spiking neurons in the guinea pig dorsal cochlear nucleus. J Comp Neurol 348:261-276.

Marks KL, Martel DT, Wu C, Basura GJ, Roberts LE, Schvartz-Leyzac KC, Shore SE (2018) Auditory-somatosensory bimodal stimulation desynchronizes brain circuitry to reduce tinnitus in guinea pigs and humans. Sci Transl Med 10:eaal3175.

McAllister BB, Dyck RH (2017) Zinc transporter 3 (ZnT3) and vesicular zinc in central nervous system function. Neurosci Biobehav Rev 80:329-350.

Mulkey RM, Malenka RC (1992) Mechanisms underlying induction of homosynaptic long-term depression in area CA1 of the hippocampus. Neuron 9:967-975.

Nakashima AS, Dyck RH (2009) Zinc and cortical plasticity. Brain Res Rev 59:347-373.

O'Connor DH, Wittenberg GM, Wang SS (2005) Dissection of bidirectional synaptic plasticity into saturable unidirectional processes. J Neurophysiol 94:1565-1573.

Oliet SH, Malenka RC, Nicoll RA (1997) Two distinct forms of long-term depression coexist in CA1 hippocampal pyramidal cells. Neuron 18:969982.

Palmiter RD, Cole TB, Quaife CJ, Findley SD (1996) ZnT-3, a putative transporter of zinc into synaptic vesicles. Proc Natl Acad Sci USA 93:1493414939.

Pan E, Zhang XA, Huang Z, Krezel A, Zhao M, Tinberg CE, Lippard SJ, McNamara JO (2011) Vesicular zinc promotes presynaptic and inhibits postsynaptic long-term potentiation of mossy fiber-CA3 synapse. Neuron 71:1116-1126.

Paoletti P, Ascher P, Neyton J (1997) High-affinity zinc inhibition of NMDA NR1-NR2A receptors. J Neurosci 17:5711-5725.

Patrick Wu HP, Dyck RH (2018) Signaling by synaptic zinc is required for whisker-mediated, fine texture discrimination. Neuroscience 369:242247.

Perez-Rosello T, Anderson CT, Schopfer FJ, Zhao Y, Gilad D, Salvatore SR, Freeman BA, Hershfinkel M, Aizenman E, Tzounopoulos T (2013) Synaptic $\mathrm{Zn}^{2+}$ inhibits neurotransmitter release by promoting endocannabinoid synthesis. J Neurosci 33:9259-9272.

Pick JE, Ziff EB (2018) Regulation of AMPA receptor trafficking and exit from the endoplasmic reticulum. Mol Cell Neurosci 91:3-9. 
Rachline J, Perin-Dureau F, Le Goff A, Neyton J, Paoletti P (2005) The micromolar zinc-binding domain on the NMDA receptor subunit NR2B. J Neurosci 25:308-317.

Rubio ME, Juiz JM (1998) Chemical anatomy of excitatory endings in the dorsal cochlear nucleus of the rat: differential synaptic distribution of aspartate aminotransferase, glutamate, and vesicular zinc. J Comp Neurol 399:341-358.

Salazar G, Love R, Werner E, Doucette MM, Cheng S, Levey A, Faundez V (2004) The zinc transporter ZnT3 interacts with AP-3 and it is preferentially targeted to a distinct synaptic vesicle subpopulation. Mol Biol Cell 15:575-587.

Salazar G, Craige B, Love R, Kalman D, Faundez V (2005) Vglut1 and ZnT3 co-targeting mechanisms regulate vesicular zinc stores in PC12 cells. J Cell Sci 118:1911-1921.

Shen W, Flajolet M, Greengard P, Surmeier DJ (2008) Dichotomous dopaminergic control of striatal synaptic plasticity. Science 321:848-851.

Snyder EM, Philpot BD, Huber KM, Dong X, Fallon JR, Bear MF (2001) Internalization of ionotropic glutamate receptors in response to mGluR activation. Nat Neurosci 4:1079-1085.

Suter BA, O’Connor T, Iyer V, Petreanu LT, Hooks BM, Kiritani T, Svoboda K, Shepherd GM (2010) Ephus: multipurpose data acquisition software for neuroscience experiments. Front Neural Circuits 4:100.

Takeda A, Fuke S, Ando M, Oku N (2009) Positive modulation of long-term potentiation at hippocampal CA1 synapses by low micromolar concentrations of zinc. Neuroscience 158:585-591.

Topolnik L, Azzi M, Morin F, Kougioumoutzakis A, Lacaille JC (2006) mGluR1/5 subtype-specific calcium signalling and induction of longterm potentiation in rat hippocampal oriens/alveus interneurones. J Physiol 575:115-131.

Tovar KR, Westbrook GL (2012) Amino-terminal ligands prolong NMDA receptor-mediated EPSCs. J Neurosci 32:8065-8073.
Traynelis SF, Wollmuth LP, McBain CJ, Menniti FS, Vance KM, Ogden KK, Hansen KB, Yuan H, Myers SJ, Dingledine R (2010) Glutamate receptor ion channels: structure, regulation, and function. Pharmacol Rev 62:405496.

Tzounopoulos T (2008) Mechanisms of synaptic plasticity in the dorsal cochlear nucleus: plasticity-induced changes that could underlie tinnitus. Am J Audiol 17:S170-S175.

Tzounopoulos T, Kim Y, Oertel D, Trussell LO (2004) Cell-specific, spike timing-dependent plasticities in the dorsal cochlear nucleus. Nat Neurosci 7:719-725.

Tzounopoulos T, Rubio ME, Keen JE, Trussell LO (2007) Coactivation of presynaptic and postsynaptic signaling mechanisms determines cell-specific spike-timing-dependent plasticity. Neuron 54:291-301.

Vergnano AM, Rebola N, Savtchenko LP, Pinheiro PS, Casado M, Kieffer BL, Rusakov DA, Mulle C, Paoletti P (2014) Zinc dynamics and action at excitatory synapses. Neuron 82:1101-1114.

Vogt K, Mellor J, Tong G, Nicoll R (2000) The actions of synaptically released zinc at hippocampal mossy fiber synapses. Neuron 26:187-196.

Wiśniewski K, Car H (2002) (S)-3,5-DHPG: a review. CNS Drug Rev 8:101116.

Wright DD, Blackstone CD, Huganir RL, Ryugo DK (1996) Immunocytochemical localization of the mGluR1 alpha metabotropic glutamate receptor in the dorsal cochlear nucleus. J Comp Neurol 364:729-745.

Wu J, Harney S, Rowan MJ, Anwyl R (2008) Involvement of group I mGluRs in LTP induced by strong high frequency stimulation in the dentate gyrus in vitro. Neurosci Lett 436:235-238.

Zhang S, Oertel D (1993) Cartwheel and superficial stellate cells of the dorsal cochlear nucleus of mice: intracellular recordings in slices. J Neurophysiol 69:1384-1397.

Zhao Y, Tzounopoulos T (2011) Physiological activation of cholinergic inputs controls associative synaptic plasticity via modulation of endocannabinoid signaling. J Neurosci 31:3158-3168. 